\title{
Factors Affecting Ultrasonic Extraction of Lead from Laboratory-Prepared Household Paint Films
}

\author{
Walter J. Rossiter, Jr. \\ Blaza Toman \\ Mary E. McKnight \\ Mana Baghai Anaraki
}

Prepared for:

U.S. Department of Housing and Urban Development Office of Healthy Homes and Lead Hazard Control 

NISTIR 6834

\title{
Factors Affecting Ultrasonic Extraction of Lead from Laboratory-Prepared Household Paint Films
}

\author{
Walter J. Rossiter, Jr. \\ Blaza Toman ${ }^{*}$ \\ Mary E. McKnight" \\ Mana Baghai Anaraki \\ *Building and Fire Research Laboratory \\ "Information Technology Laboratory \\ National Institute of Standards and Technology \\ Gaithersburg, MD 20899-8621
}

May 2002
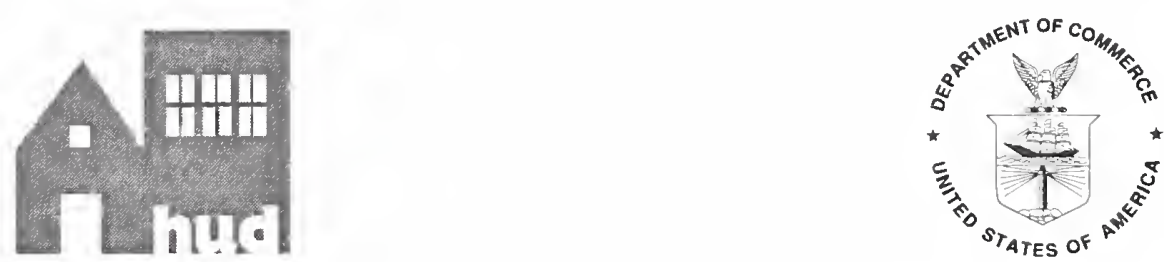

Prepared for:

U.S. Department of Housing and Urban Development
Mel Martine- Secretary

Office of Healthy Homes and Lead Hazard Control David E. Jacobs, Director
U.S. Department of Commerce Donald L. Evans, Secretary

Technology Administration Phillip J. Bond, Under Secretary for Technology

National Institute of Standards and Technology Arden L. Bement, Jr., Director 



\begin{abstract}
In a previous National Institute of Standards and Technology (NIST) study on the reliability of ultrasonic extraction-anodic stripping voltammetry (UE/ASV) for quantitatively determining lead in paint films, it was found that the amount of lead was often considerably less than the known lead levels of the specimens. An important contributor appeared to be incomplete lead solubilization during ultrasonication. This report presents the results of a follow-up study performed to examine factors affecting ultrasonic extraction of lead from laboratory-prepared paint films that had been characterized using common analytical methods. The current study had three phases. In Phase I, five experimental variables—sonicator power, specimen mass, specimen particle size, sonication temperature, and sonication time-were systematically examined in a controlled two-level experiment. Three significant main effects—-particle size, temperature, and time-were identified. Two significant two-way interactions-particle size with temperature and particle size with time-were also observed. No three-way interactions were found. The effect of particle size was strong. When the particle size was small $(\leq 425 \mu \mathrm{m})$, mean lead recovery was quantitative regardless of the conditions of sonication time and temperature. In contrast, when the particle size was large, only in the case of relatively high temperature $\left(\approx 65^{\circ} \mathrm{C}\right)$ and long time $(90 \mathrm{~min})$ was the mean recovery quantitative (i.e., $80 \%$ and above). In Phase II, ultrasonic extractions, conducted under temperature and time conditions found in Phase I to be most effective, were performed on specimens sampled from each of the 80 NIST paint-film panels. Lead recoveries were higher than the mean recoveries reported in the previous NIST UE/ASV study for each panel. In Phase II, lead extractions from specimens sampled from a limited number of NIST paint-film panels were performed without ultrasound using a water bath with mechanical stirring of the specimens in acid solution. The results were compared with those obtained when extraction was conducted using a sonicator. Lead recoveries with and without ultrasound were comparable for the same conditions of temperature and time. In conducting UE/ASV analysis of paint-film samples, small particle size of the ground specimen needs to be maintained.
\end{abstract}

Key Words: analysis; anodic stripping voltammetry (ASV); building technology; lead-based paint; lead recovery; particle size; sonicator power; sonication temperature; sonication time; testing; ultrasonic extraction (UE) 


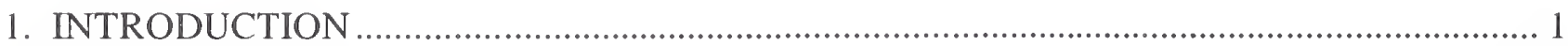

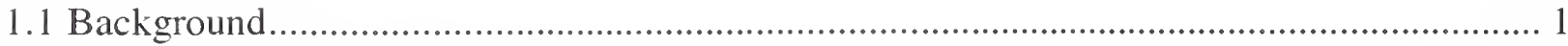

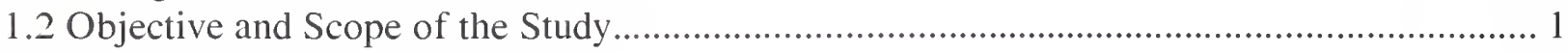

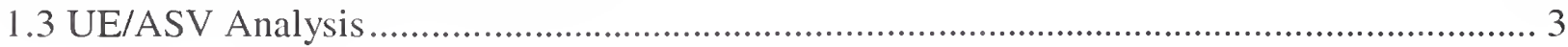

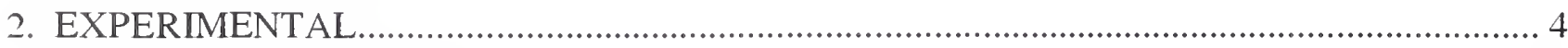

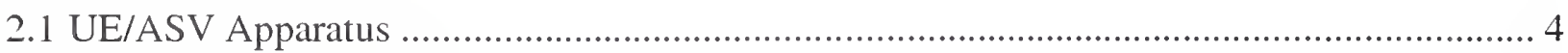

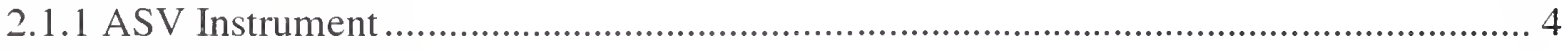

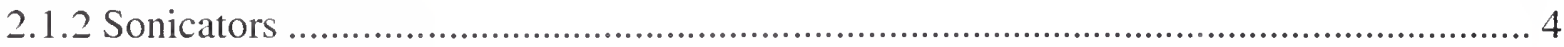

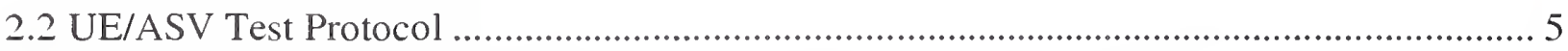

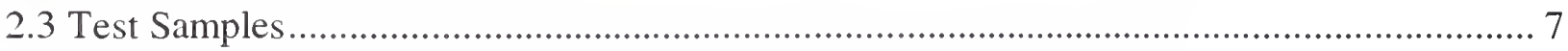

2.3.1 NIST Paint-Film Panels, and Specimen Mass and Particle Size .................................... 7

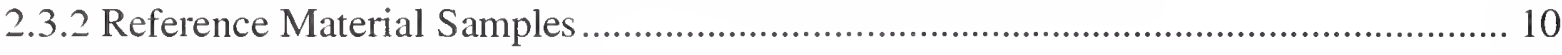

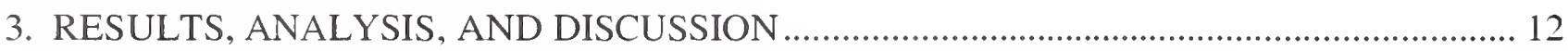

3.1 Factors Affecting UE Extraction from Laboratory-Prepared Paint Films .............................. 12

3.1.1 Preliminary Experiment Including Sonicator Effect ....................................................... 12

3.1.2 Five-Factor Experiment …………............................................................................ 15

3.1.3 Comparison of ASV Results with ICP Results ............................................................ 23

3.2 Lead Extraction from the Series of NIST Paint-Film Panels ................................................. 25

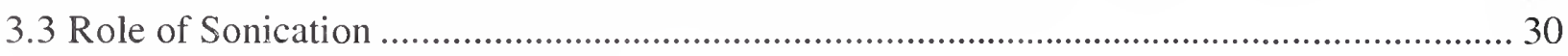

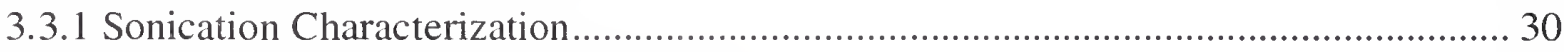

3.3.2 Lead Extraction Without Ultasound .............................................................................. 33

3.3.3 SEM Comparison of Specimens Extracted With and Without Sonication ................... 35

3.4 Proposed Conditions for Lead Extraction in the Field ........................................................... 35

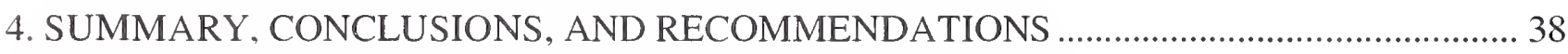

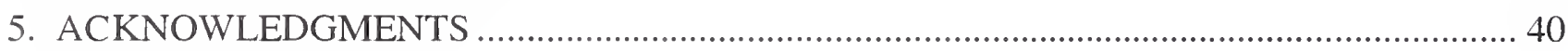

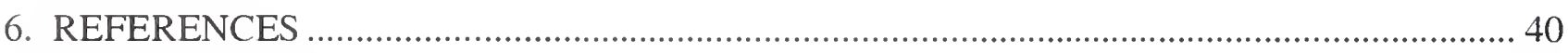




\section{LIST OF TABLES}

$\underline{\text { Page }}$

Table 1. Experimental variables examined in the previous NIST study 2

Table 2. Experimental variables examined in a two-level experiment investigating factors affecting ultrasonic extraction of lead from laboratory-prepared paint films....................... 3

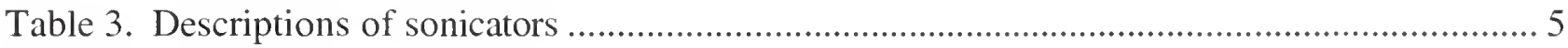

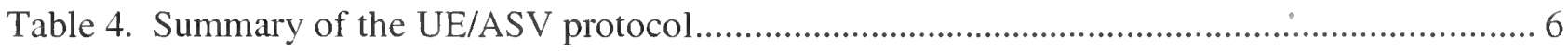

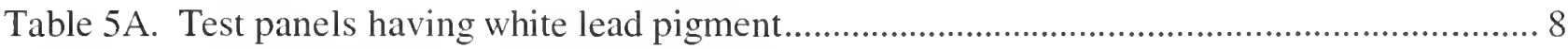

Table 5B. Test panels having lead chromate pigment ................................................................ 9

Table 6. Particle size breakdown of ground paint films sampled from selected paint-film panels.. 10

Table 7. Preparation details for specimens designated as low or high mass, and small................... 11

Table 8. Mass measurements as a related to specimen mass and particle size................................. 11

Table 9. Variables examined in the preliminary experiment investigating factors affecting ........... 13

Table 10. Lead recovery determined in the five-factor, two-level experiment ................................ 16

Table 11. Analysis of variance on the log recovery for the five-factor experiment........................ 19

Table 12A. UE/ASV lead recovery from the NIST paint-film panels determined using high sonication temperature and long sonication time for test panels containing white lead pigment. The results are compared with those of the previous NIST study........ 27

Table 12B. UE/ASV lead recovery from the NIST paint-film panels determined using high sonication temperature and long sonication time for test panels containing lead chromate pigment. The results are compared with those of the previous NIST study. 28

Table 13. Analysis of variance on the log lead recovery for the ultrasonic extraction 30

Table 14. Paint-film panels for the experiment extracting lead without ultrasound using a water bath with mechanical stirring of the specimen in the acid solution.

Table 15. Comparison of lead recovery for extractions performed with and without ultrasound... 34

Table 16. Recommendations for extraction temperature and time as related to the particle size of ground laboratory-prepared paint-film specimens 36 


\section{LIST OF FIGURES}

Figure 1. Lead Recovery Versus Sonicator Power.

Figure 2. Lead Recovery Versus Sonication Time As Determined at Low and High Temperature for Specimens Sampled from Panel 127

Figure 3. Lead Recovery Versus Combination of Experimental Variables

Figure 4. Box plots of lead recovery as a function of: (A) particle size, (B) sonication temperature, and $(\mathrm{C})$ sonication time

Figure 5. The interaction between particle size and (A) temperature, and (B) time, respectively ... 21

Figure 6. Lead Recovery for the Combinations of Specimen Particle Size, Sonication Time, and Sonication Temperature

Figure 7. Comparison of the Results of Lead Measurements Performed on Extract Solutions Using ASV and ICP Methods

Figure 8. UE/ASV Measured Lead Result Versus Known Panel Lead Level 26

Figure 9. Parametric Effects on Lead Recovery.

Figure 10. Maximum Temperatures $\left({ }^{\circ} \mathrm{C}\right)$ in Each of the Seven Sonicator Tubes Placed in Sonicator No. 1

Figure 11. Lead Recovery from Specimens Sampled from Panel 102 Versus Sonication Tube Temperature

Figure 12. Specimen Mass Passing a Sieve as a Function of Sieve Mesh 36 


\section{INTRODUCTION \\ 1.1 Background}

Field-portable ultrasonic extraction-anodic stripping voltammetry (UE/ASV) has been considered an attractive analytical technology for quantitatively determining the amount of lead in environmental samples such as paint and airborne particles. This technology provides relative ease of operation, rapid on-site response, and potentially acceptable cost. Commercial UE/ASV apparatus $^{*}$ designed for field analysis of lead in paint is available in the United States. Over the last decade, a number of laboratory and field studies [1-6] have been performed to evaluate the reliability of field-portable UE/ASV for measurement of lead in paint. The results have indicated that lead recoveries from paint and related samples have, for the most part, ranged from $75 \%$ to complete (i.e., quantitative) recovery. Consequently, the authors have generally concluded that UE/ASV may be suitable for conducting extensive quantitative on-site testing of lead in household paint.

In 2001, the National Institute of Standards and Technology (NIST) completed a study, sponsored by the U.S. Department of Housing and Urban Development (HUD), on the reliability of fieldportable UE/ASV for quantitatively determining the lead level of laboratory-prepared paint films when certified lead inspectors trained to conduct UE/ASV tests performed the analyses [7]. The NIST study examined the effect of six experimental variables (Table 1) on lead recovery. The test samples were 80 paint-film panels that had been prepared in the laboratory to control factors including lead level, lead pigment type, paint-film substrate, and the thickness and type of overlayer paint coated on the lead-containing film. A major finding was that lead levels determined according to the UE/ASV protocol were often considerably less than the known test panel lead levels.

Depending on the combination of experimental factors-operator, lead pigment type, substrate type, overlayer, and apparatus-lead recovery ranged from $28 \%$ to $94 \%$, with a median of $63 \%$. These findings were in notable contrast with previously published lead recoveries which, as indicated above, generally ranged from $75 \%$ to quantitative recovery.

Extensive investigations were not conducted during the NIST UE/ASV study [7] to examine why the lead recoveries were, in many cases, considerably less than the lead levels of the test panels. Based on quality assurance measurements made using standardized aqueous lead solutions, ASV measurement error did not appear to play a role. On the other hand, a key contributor appeared to be incomplete lead solubilization during paint specimen sonication. Analysis of a limited number of paint-film residues remaining in the sonicator tubes after specimen sonication found lead in amounts ranging from about $5 \%$ to $58 \%$ of the lead levels of the test panels. Questions were consequently raised as to whether specimen sonication, specimen grinding before sonication, or interaction between these two factors played a role in the incomplete lead solubilization. Understanding why the UE method used in the NIST UE/ASV study resulted in low lead recovery is obviously important for practical use of ASV in the field. Unlike UE, other common extraction methods such as hotplate or microwave acid digestion are not suitable for routine field use [1].

\subsection{Objective and Scope of the Study}

This report presents the results of a HUD-sponsored study to examine factors affecting the ultrasonic extraction of lead from laboratory-prepared paint specimens. The results are intended to improve protocols for using UE in the field.

\footnotetext{
*For an overview of the basic procedure used in an UE/ASV analysis, the reader is referred to Section 1.3.
} 


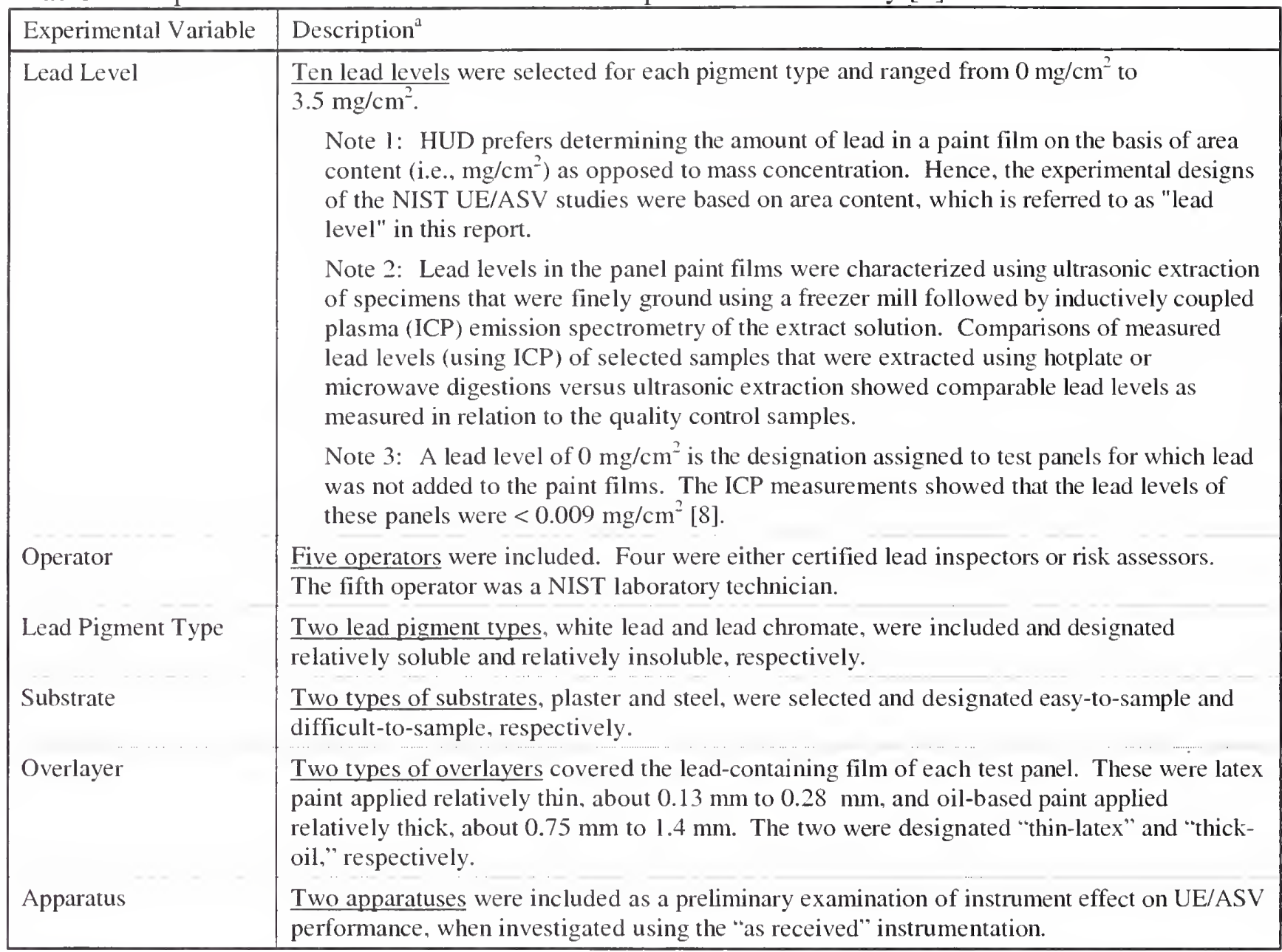

${ }^{a}$ The information summarized in this table is taken from NISTIR 657l [7] to which the reader is referred for a more complete description of each factor and commentary as to why each was incorporated in the previous NIST study.

The study had three phases that were, for the most part, performed sequentially:

- In Phase I, five experimental variables (Table 2)—sonicator power, specimen mass, specimen particle size, sonication temperature, and sonication time-that might potentially affect ultrasonic extraction of lead from laboratory-prepared paint films were systematically varied in a controlled two-level experiment (See Section 3.1).

- In Phase II, ultrasonic extractions, conducted under sonication temperature and time conditions found in Phase I to enhance lead recovery, were performed on specimens sampled from each of the 80 NIST paint-film panels. The amount of lead extracted was determined using ASV (See Section 3.2).

- In Phase III, lead extractions from specimens sampled from a limited number of NIST paintfilm panels and from reference materials were performed without ultrasound using a water bath with mechanical stirring of the specimen in the acid solution. The results were compared with those obtained when extraction was conducted using a sonicator under the same temperature and time conditions (See Section 3.3). 
Table 2. Experimental variables examined in a two-level experiment investigating factors affecting ultrasonic extraction of lead from laboratory-prepared paint films

\begin{tabular}{|c|c|}
\hline Variable & Designated Level \\
\hline Sonicator Power & $\begin{array}{l}\text { - Low power }(45 \mathrm{~W} \text { using Sonicator No. } 1)^{\mathrm{a}} \\
\text { - High power }(600 \mathrm{~W} \text { using Sonicator No. } 9)^{\mathrm{a}}\end{array}$ \\
\hline Specimen Mass & $\begin{array}{l}\text { - Low mass }(\approx 25 \mathrm{mg}) \\
\text { - } \quad \text { High mass }(\approx 100 \mathrm{mg})\end{array}$ \\
\hline \multirow[t]{2}{*}{ Specimen Particle Size } & $\begin{array}{l}\text { - Small particles }(\text { all }<425 \mu \mathrm{m} \text { with the majority }<180 \mu \mathrm{m})^{\mathrm{b}} \\
\text { - Large particles }(\text { majority }>425 \mu \mathrm{m})^{\mathrm{b}}\end{array}$ \\
\hline & $\begin{array}{l}\text { Note: Small particles resulted from grinding specimens mechanically } \\
\text { in a freezer mill at liquid nitrogen temperatures; whereas large } \\
\text { particles were obtained by grinding specimens manually (using the } \\
\text { common UE/ASV technique) after cooling in dry ice. }\end{array}$ \\
\hline Sonication Temperature & $\begin{array}{l}\text { - Low temperature }\left(\approx 42{ }^{\circ} \mathrm{C} \text { to } 47^{\circ} \mathrm{C}\right) \\
\text { - High temperature }\left(\approx 62{ }^{\circ} \mathrm{C} \text { to } 67^{\circ} \mathrm{C}\right)\end{array}$ \\
\hline Sonication Time & $\begin{array}{l}\text { - Short time }(30 \mathrm{~min}) \\
\text { - Long time }(90 \mathrm{~min})\end{array}$ \\
\hline $\begin{array}{l}\text { NOTE: In the previous } \\
\text { the following experimen }\end{array}$ & $\begin{array}{l}\text { UE/ASV study [7], the sonication extraction conditions corresponded to } \\
\text { ariables: low sonicator power, high specimen mass, large specimen }\end{array}$ \\
\hline
\end{tabular}

${ }^{4}$ Sonicators are described in Table 3.

${ }^{\mathrm{b}}$ See Section 2.3.1.

\subsection{UE/ASV Analysis}

The basic procedure for using the UE/ASV apparatus has two main steps. First, the paint specimen is removed from its substrate, ground, and subjected to ultrasonic extraction (UE) with nitric acid. Second, the lead concentration of the extraction solution is determined using anodic stripping voltammetry (ASV). A brief description of the principle of ASV analysis of an analyte metal species dissolved in solution (e.g., $\mathrm{Pb}^{+2}$ ion) is given in ASTM Standard Guide E 1775, "Evaluating Performance of On-Site Extraction and Field-Portable Electrochemical or Spectrophotometric Analysis for Lead" [9]:

"The analyte is first deposited (preconcentrated) electrochemically by reducing the dissolved ion in solution to immobilized metal species at a mercury electrode surface. The metal is deposited in the form of an amalgam (with $\mathrm{Hg}$ ) at an applied potential (voltage) which is negative of the standard oxidation potential for the metal/ion redox couple. After deposition, the preconcentrated metal species is then "stripped" from the mercury electrode by applying a positive potential sweep, which causes anodic oxidation of the analyte metal species to dissolved ion. The current associated with this reoxidation is measured. The peak current is proportional to the original concentration of dissolved analyte species over a wide range of concentrations." 
A commercial, field-portable UE/ASV apparatus" was the main instrumentation used in the study. It was purchased for use in the previous NIST study, in which it was designated Apparatus 1 [7].

2.1.1 ASV Instrument. The field-portable ASV instrument is battery operated, uses disposable electrodes, and is factory-calibrated. Results of a lead analysis for paint specimens are given in units of either "mg/cm" or "mg." The limit of detection for lead reported in the supplier"s instruction booklet is $0.04 \mathrm{mg} / \mathrm{cm}^{2}$ or $0.02 \mathrm{mg}$. Factory calibration was checked in the previous NIST UE/ASV study using six standard solutions of lead nitrate in $2.5 \%$ nitric acid (volume fraction ) [7]. Because the results of the lead analyses of the test panels were generally recorded in " $\mathrm{mg} / \mathrm{cm}^{2}$," the calibration checks were conducted in units of $\mathrm{mg} / \mathrm{cm}^{2}$. The six standard solutions ( $\mathrm{Pb}$ contents ranging from $1 \mathrm{mg} / \mathrm{L}$ to $50 \mathrm{mg} / \mathrm{L}$ ) covered a concentration range that corresponded to an ASV range of $0.1 \mathrm{mg} / \mathrm{cm}^{2}$ to $5 \mathrm{mg} / \mathrm{cm}^{2}$, which was the lead level range in the NIST paint-film panels. A repeat calibration check performed at the beginning of the current study using six standard lead nitrate solutions confirmed that the instrument remained in calibration; the ASV response versus lead concentration was linear $\left(r^{2}=0.999\right)$. In addition, two calibration checks were normally performed at the beginning and end of each day's analyses using one of the six standard solutions of lead nitrate in $2.5 \%$ nitric acid. These checks indicated that (1) the ASV instrument remained in calibration at the beginning of the testing, and (2) instrument drift over the course of testing did not occur. Exception to two calibration checks per day was made on those days when only a few UE/ASV analyses (normally less than 6) were performed. In these cases, a single calibration check was carried out.

2.1.2 Sonicators. Nine sonicators were included in this study. Table 3 summarizes their dimensions and power as taken from manufacturers' literature. Sonicator Nos. 1 and 2 belonged to NIST; the others were either used in the NIST laboratories on loan from manufacturers or in laboratories of other researchers who had experience with UE/ASV analysis of paints or other environmental samples. Sonicator No. 1 was supplied with the UE/ASV apparatus described in Section 2.1. A set of seven specimens can be simultaneously extracted using this sonicator. Although the baths of some sonicators were large enough to accommodate more than seven specimens simultaneously, this was not done.

The sonicator-operation instructions provided by the UE/ASV supplier include a performance check for whether the sonicator is operating acceptably. The procedure is also given in Appendix A of Grohse, Gutknecht, Luk, Wilson, and Van Hise [10]. In conducting the performance check, the sonicator bath is filled with tap water, at $\approx 50{ }^{\circ} \mathrm{C}$, to which two or three drops of a detergent are added. After running the sonicator for $5 \mathrm{~min}$ (to degas the bath), a sheet of thin aluminum foil $(\approx 0.03 \mathrm{~mm}$ thick $)$ is placed in the bottom center of the bath. The sonicator is operated for $45 \mathrm{~s}$, after which time the foil is examined for small indentations and perforations. If the foil is unaffected by the sonication, then the sonicator is considered to be operating incorrectly. The sonicators produced numerous small indentations and perforations in the aluminum foil that were observable almost immediately after sonication began.

\footnotetext{
"In this report, the term, "apparatus," refers to the combination of UE sonicator and ASV electrochemical instrument that was purchased as part of a field-portable kit. For the individual pieces of equipment, the terms, "[UE] sonicator" and "[ASV] instrument" are used.
} 
Table 3. Descriptions of sonicators ${ }^{a}$

\begin{tabular}{|c|c|c|c|c|c|}
\hline$\frac{\text { Sonic. }}{\text { No. }}$ & $\frac{\text { Bath Dimension }^{\mathrm{b}}}{\mathrm{cm} \times \mathrm{cm} \times \mathrm{cm}}$ & $\frac{\text { Area }}{\mathrm{cm}^{2}}$ & $\frac{\text { Volume }}{\mathrm{L}}$ & $\frac{\text { Power }}{W}$ & $\frac{\text { Power Density }}{\mathrm{W} / \mathrm{cm}^{2}}$ \\
\hline 1 & $14.6 \times 13.3 \times 10.2$ & 194 & 1.9 & 45 & 0.23 \\
\hline 2 & $24.1 \times 14.0 \times 10.2$ & 337 & 2.8 & 100 & 0.30 \\
\hline 3 & $29.2 \times 24.1 \times 15.2$ & 705 & 9.5 & 135 & 0.19 \\
\hline 4 & $49.5 \times 29.2 \times 15.2$ & 1445 & 21 & 270 & 0.19 \\
\hline 5 & $30.5 \times 45.7 \times 33.0$ & 1394 & 42 & $833^{d}$ & 0.60 \\
\hline 6 & $17.5 \times 25.0 \times 25.0$ & 438 & 11 & 250 & 0.57 \\
\hline 7 & $29.2 \times 15.2 \times 15.2$ & 444 & 5.7 & 100 & 0.23 \\
\hline 8 & $15.2 \times 14.0 \times 10.2$ & 213 & 1.9 & 70 & 0.33 \\
\hline 9 & $30.5 \times 30.5 \times 22.5$ & 930 & 21 & 600 & 0.64 \\
\hline
\end{tabular}

'This information is taken from manufacturers' literature.

${ }^{b}$ Dimensions are listed in order of length, width, and depth.

${ }^{c}$ Values were estimated by dividing sonicator power by the bath surface area.

${ }^{\mathrm{d}}$ Sonicator No. 5 was specified as having $1000 \mathrm{~W}$ power, but it was not operated at full power.

\subsection{UE/ASV Test Protocol}

The UE/ASV analyses were conducted according to the test protocol (Table 4) developed for the previous NIST study according to the instructions provided by the UE/ASV supplier for use of its apparatus [7]. Williams, Van Hise, and Gutknecht [2] have commented that the UE/ASV supplier's instructions for specimen grinding and sonicator operation are based on EPA ${ }^{*}$ recommendations for ultrasonic extraction, as first suggested by Luk, Grohse, Hodson, Binstock, Van Hise, and Gutknecht [11]. It is noted herein that the supplier's instructions indicate that grinding is performed "until all large particles are broken down." In contrast, Luk et al. [11] state that, in preparing the specimen, "grind [the paint] until a fine powder is achieved." Similarly, ASTM Standard Practice E 1979, "Ultrasonic Extraction of Paint, Soil, and Air Samples for Subsequent Determination of Lead," directs that, when preparing a paint sample for ultrasonic extraction, "... grind and homogenize the ... paint sample to a fine powder" [12]

Exceptions to the NIST protocol (Table 4) in the current study involved those tests where the specimen preparation and sonication conditions were altered in investigating factors affecting lead extraction using sonication. Note that, in following the NIST protocol, all paint-film specimens are cooled using dry ice prior to grinding. This is a key deviation from the supplier's instructions, but was incorporated in the protocol because dry-ice cooling had been reported by Ashley et al. [3] to assist significantly the grinding of paint-film specimens prior to sonication. ASTM E 1979 [12] also states that "the use of dry ice assists greatly in the grinding..."

When conducting the lead extractions, if the sonicator was not equipped with temperature control, the sonication temperature was maintained for a 30 min extraction by filling the bath with preheated water at the assigned temperature (i.e., $\approx 45^{\circ} \mathrm{C}$ or $65^{\circ} \mathrm{C}$ ). When the extraction time was $90 \mathrm{~min}$, sonication was briefly stopped after each of the first two 30 min periods (because the water had

*US Environmental Protection Agency. 


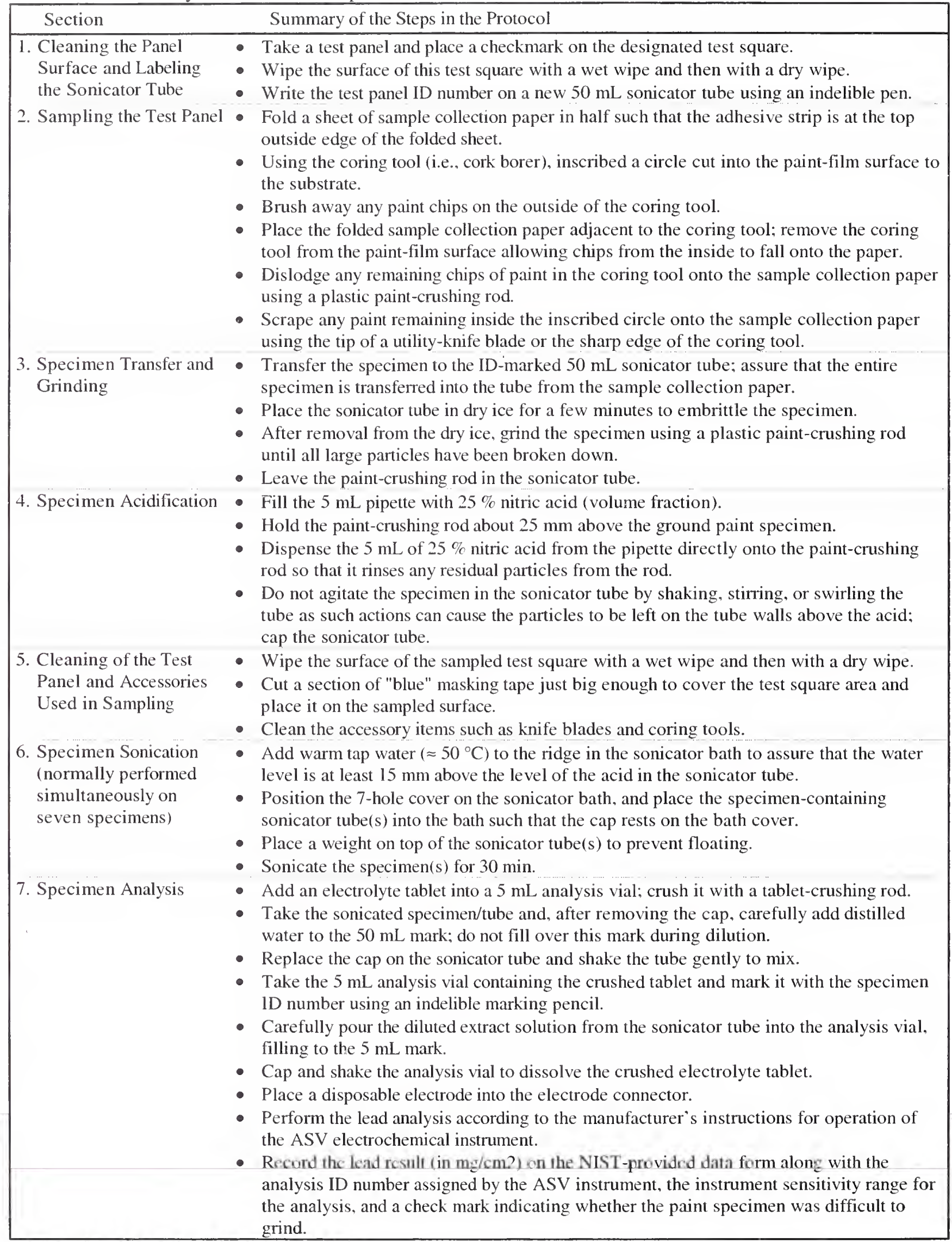

\footnotetext{
This table is taken from NISTIR 6571 [7].
} 
slightly cooled) during which the bath was partially emptied and re-filled with preheated water at the assigned temperature.

\subsection{Test Samples}

2.3.1 NIST Paint-Film Panels, and Specimen Mass and Particle Size. Tables 5A and 5B describe the test panels having white lead and lead chromate pigments, respectively. These panels were originally fabricated in the laboratory for use in a HUD-sponsored study on the performance of spot test kits [8]. Each panel included a uniformly thick, lead-containing paint layer for which the lead level had been determined using common laboratory methods of analysis. The use of these panels precluded independent investigation of the effect of overlayer type and overlayer thickness on UE/ASV response. Note in Table 1 that latex paint overlayers were thin; whereas oil paint overlayers were thick. Test specimens were randomly sampled from a $25 \mathrm{~mm}$ by $25 \mathrm{~mm}$ square grid indelibly imprinted on the surface of each panel.

Specimens designated as having "large particle size" were manually ground according to the steps in the test protocol after cooling them in dry ice (Table 4; Section 3). Specimens having "small particle size" were mechanically ground for about $5 \mathrm{~min}$ at liquid nitrogen temperatures using a freezer mill (Spex Model 6700\%). When using the freezer mill, multiple samples taken from a single paint-film panel were ground simultaneously. It was assumed that the lead was homogeneously distributed in the resultant composite sample of small particle test material. Test specimens were obtained from the freezer-mill ground composite using a laboratory spatula.

To estimate the particle size of manually ground and freezer-mill ground specimens, sieve analysis was conducted on selected specimens (Table 6). The five sieves (Nos. 10, 20, 40, 80, and 100) conformed to ASTM Standard Specification E 11 [13] and have mesh sizes of $2000 \mu \mathrm{m}, 850 \mu \mathrm{m}$, $425 \mu \mathrm{m}, 180 \mu \mathrm{m}$, and $150 \mu \mathrm{m}$, respectively. The "small particle" specimen consisted of particles that were less than $425 \mu \mathrm{m}$ with the majority $(\approx 60 \%)$ being less than $180 \mu \mathrm{m}$. In contrast, the "large particle" specimens had particles that were, for the most part, greater than $425 \mu \mathrm{m}$ with the majority being greater than $850 \mu \mathrm{m}$. To define the area of a specimen removed from a paint-film panel, either a $4.0 \mathrm{~mm}$ (inside diameter) No. 1 or $7.9 \mathrm{~mm}$ No. 4 cork borer ${ }^{\dagger}$ was used. Their areas were $13 \mathrm{~mm}^{2}$ and $49 \mathrm{~mm}^{2}$, respectively, and the resultant specimens were designated as being of "low" or "high" mass, respectively. Specimen mass was determined using an analytical balance (Mettler Model AT201) that weighed to $0.01 \mathrm{mg}$.

Panel 127 was selected for use in the preliminary experiment conducted in designing the five-factor experiment investigating variables affecting ultrasonic extraction of lead from the laboratory paintfilm panels. This panel was considered suitable because the substrate was plaster (i.e., easy to sample), the lead pigment was white lead (i.e., relatively easy to extract versus lead chromate), and the lead level was $3.88 \mathrm{mg} / \mathrm{cm}^{2}$ (i.e., relatively high). Additionally, in the previous NIST UE/ASV study [7], the mean lead recovery for 10 analyses performed on specimens from this panel was $24 \%$, with values ranging from $11 \%$ to $44 \%$. Thus, improvement in lead recovery resulting from changes in the experimental variable should be readily discernible. During preliminary testing, two replicate specimens from the panel were generally analyzed for each of the factors investigated.

\footnotetext{
Certain trade names or company products are mentioned in the text to specify adequately the experimental procedure and equipment used. In no case does such identification imply recommendation or endorsement by the National Institute of Standards and Technology, nor does it imply that the equipment is the best available for the purpose.

${ }^{\top}$ A No. 4 cork borer is the paint sampling tool supplied with the UE/ASV apparatus.
} 
Table 5A. Test panels having white lead pigment

\begin{tabular}{|c|c|c|c|c|}
\hline \multirow{3}{*}{$\begin{array}{c}\text { Test } \\
\text { Panel } \\
\text { ID Code }\end{array}$} & \multicolumn{4}{|c|}{ Panel Description } \\
\hline & \multirow{2}{*}{$\begin{array}{c}\text { Substrate } \\
\text { Type }\end{array}$} & \multicolumn{2}{|c|}{ Overlayer } & \multirow{2}{*}{$\frac{\text { Lead Level }^{\mathrm{b}, \mathrm{c}, \mathrm{d}}}{\mathrm{mg} / \mathrm{cm}^{2}}$} \\
\hline & & Type & Thickness ${ }^{a}$ & \\
\hline 107 & Plaster & Latex & Thin & $<0.009$ \\
\hline 313 & Plaster & Oil & Thick & $<0.009$ \\
\hline 188 & Plaster & Latex & Thin & 0.10 \\
\hline 285 & Plaster & Oil & Thick & 0.09 \\
\hline 234 & Plaster & Latex & Thin & 0.19 \\
\hline 237 & Plaster & Oil & Thick & 0.19 \\
\hline 165 & Plaster & Latex & Thin & 0.28 \\
\hline 138 & Plaster & Oil & Thick & 0.31 \\
\hline 262 & Plaster & Latex & Thin & 0.39 \\
\hline 129 & Plaster & Oil & Thick & 0.43 \\
\hline 206 & Plaster & Latex & Thin & 0.48 \\
\hline 281 & Plaster & Oil & Thick & 0.50 \\
\hline 179 & Plaster & Latex & Thin & 0.70 \\
\hline 292 & Plaster & Oil & Thick & 0.66 \\
\hline 256 & Plaster & Latex & Thin & 1.04 \\
\hline 102 & Plaster & Oil & Thick & 0.96 \\
\hline 251 & Plaster & Latex & Thin & 1.53 \\
\hline 279 & Plaster & Oil & Thick & 1.54 \\
\hline 177 & Plaster & Latex & Thin & 3.53 \\
\hline 127 & Plaster & Oil & Thick & 3.88 \\
\hline 322 & Steel & Latex & Thin & $<0.009$ \\
\hline 101 & Steel & Oil & Thick & $<0.009$ \\
\hline 221 & Steel & Latex & Thin & 0.09 \\
\hline 168 & Steel & Oil & Thick & 0.09 \\
\hline 269 & Steel & Latex & Thin & 0.17 \\
\hline 180 & Steel & Oil & Thick & 0.18 \\
\hline 203 & Steel & Latex & Thin & 0.23 \\
\hline 135 & Steel & Oil & Thick & 0.23 \\
\hline 222 & Steel & Latex & Thin & 0.32 \\
\hline 189 & Steel & Oil & Thick & 0.29 \\
\hline 326 & Steel & Latex & Thin & 0.44 \\
\hline 130 & Steel & Oil & Thick & 0.36 \\
\hline 224 & Steel & Latex & Thin & 0.58 \\
\hline 226 & Steel & Oil & Thick & 0.54 \\
\hline 278 & Steel & Latex & Thin & 0.78 \\
\hline 246 & Steel & Oil & Thick & 0.86 \\
\hline 284 & Steel & Latex & Thin & 1.39 \\
\hline 123 & Steel & Oil & Thick & 1.46 \\
\hline 215 & Steel & Latex & Thin & 3.20 \\
\hline 145 & Steel & Oil & Thick & 3.39 \\
\hline
\end{tabular}

a Thin range: $0.13 \mathrm{~mm}$ to $0.28 \mathrm{~mm}$; thick range: $0.75 \mathrm{~mm}$ to $1.4 \mathrm{~mm}$.

${ }^{\mathrm{b}}$ A lead level of $0 \mathrm{mg} / \mathrm{cm}^{2}$ is the designation assigned to test panels for which lead was not added to the paint films. Measurements using inductively coupled plasma (ICP) emission spectrometry showed that the lead level for these panels was $<0.009 \mathrm{mg} / \mathrm{cm}^{2}$.

${ }^{c}$ A distinction between white lead and lead chromate for specimens having a $0 \mathrm{mg} / \mathrm{cm}^{2}$ lead level is artificial because such specimens did not have added lead. Nevertheless, the distinction is maintained to balance the experimental design.

${ }^{\mathrm{d}}$ The measurement process used in assigning the values is given in Ref. [8]. 
Table 5B. Test panels having lead chromate pigment

\begin{tabular}{|c|c|c|c|c|}
\hline \multirow{3}{*}{$\begin{array}{c}\text { Test } \\
\text { Panel } \\
\text { ID Code }\end{array}$} & \multicolumn{4}{|c|}{ Panel Description } \\
\hline & \multirow{2}{*}{$\begin{array}{c}\text { Substrate } \\
\text { Type }\end{array}$} & \multicolumn{2}{|c|}{ Overlayer } & \multirow{2}{*}{$\frac{\text { Lead Level }^{\mathrm{b}, \mathrm{c}, \mathrm{d}}}{\mathrm{mg} / \mathrm{cm}^{2}}$} \\
\hline & & Type & Thickness $^{a}$ & \\
\hline 175 & Plaster & Latex & Thin & $<0.009$ \\
\hline 202 & Plaster & Oil & Thick & $<0.009$ \\
\hline 250 & Plaster & Latex & Thin & 0.47 \\
\hline 140 & Plaster & Oil & Thick & 0.51 \\
\hline 274 & Plaster & Latex & Thin & 0.69 \\
\hline 163 & Plaster & Oil & Thick & 0.74 \\
\hline 323 & Plaster & Latex & Thin & 0.94 \\
\hline 297 & Plaster & Oil & Thick & 0.94 \\
\hline 126 & Plaster & Latex & Thin & 1.09 \\
\hline 178 & Plaster & Oil & Thick & 1.20 \\
\hline 148 & Plaster & Latex & Thin & 1.46 \\
\hline 183 & Plaster & Oil & Thick & 1.44 \\
\hline 249 & Plaster & Latex & Thin & 1.71 \\
\hline 253 & Plaster & Oil & Thick & 1.62 \\
\hline 139 & Plaster & Latex & Thin & 1.98 \\
\hline 141 & Plaster & Oil & Thick & 1.86 \\
\hline 333 & Plaster & Latex & Thin & 2.51 \\
\hline 330 & Plaster & Oil & Thick & 2.30 \\
\hline 167 & Plaster & Latex & Thin & 3.29 \\
\hline 205 & Plaster & Oil & Thick & 3.64 \\
\hline 303 & Steel & Latex & Thin & $<0.009$ \\
\hline 319 & Steel & Oil & Thick & $<0.009$ \\
\hline 223 & Steel & Latex & Thin & 0.49 \\
\hline 308 & Steel & Oil & Thick & 0.43 \\
\hline 289 & Steel & Latex & Thin & 0.57 \\
\hline 310 & Steel & Oil & Thick & 0.52 \\
\hline 216 & Steel & Latex & Thin & 0.74 \\
\hline 302 & Steel & Oil & Thick & 0.75 \\
\hline 231 & Steel & Latex & Thin & 0.98 \\
\hline 113 & Steel & Oil & Thick & 0.92 \\
\hline 158 & Steel & Latex & Thin & 1.34 \\
\hline 124 & Steel & Oil & Thick & 1.48 \\
\hline 187 & Steel & Latex & Thin & 1.55 \\
\hline 315 & Steel & Oil & Thick & 1.57 \\
\hline 263 & Steel & Latex & Thin & 1.56 \\
\hline 294 & Steel & Oil & Thick & 1.89 \\
\hline 329 & Steel & Latex & Thin & 1.72 \\
\hline 331 & Steel & Oil & Thick & 2.25 \\
\hline 199 & Steel & Latex & Thin & 3.09 \\
\hline 142 & Steel & Oil & Thick & 2.81 \\
\hline
\end{tabular}

Thin range: $0.13 \mathrm{~mm}$ to $0.28 \mathrm{~mm}$; thick range: $0.75 \mathrm{~mm}$ to $1.4 \mathrm{~mm}$.

${ }^{\mathrm{b}}$ A lead level of $0 \mathrm{mg} / \mathrm{cm}^{2}$ is the designation assigned to test panels for which lead was not added to the paint films. Measurements using inductively coupled plasma (ICP) emission spectrometry showed that the lead level for these panels was $<0.009 \mathrm{mg} / \mathrm{cm}^{2}$.

${ }^{\mathrm{c}}$ A distinction between white lead and lead chromate for specimens having a $0 \mathrm{mg} / \mathrm{cm}^{2}$ lead level is artificial because such specinmens did not have added lead. Nevertheless, the distinction is maintained to balance the experimental design.

${ }^{\mathrm{d}}$ The measurement process used in assigning the values is given in Ref. [8]. 
Table 6. Particle size breakdown of ground paint films sampled from selected paint-film panels

\begin{tabular}{|c|c|c|c|c|c|c|c|}
\hline \multirow[b]{2}{*}{$\begin{array}{c}\text { Particle Size Breakdown } \\
\text { Using Sieve Analysis }\end{array}$} & \multicolumn{3}{|c|}{ Panel 127-MG } & \multicolumn{3}{|c|}{ Panel $179-\mathrm{MG}^{\mathrm{b}}$} & \multirow{2}{*}{$\begin{array}{c}\frac{\text { Panel } 279-\mathrm{FG}^{\mathrm{c}}}{\text { Single Measure. }}{ }^{\mathrm{g}} \\
\%\end{array}$} \\
\hline & $\begin{array}{c}\operatorname{Mean}^{\mathrm{d}} \\
\%\end{array}$ & $\begin{array}{c}\mathrm{SD}^{\mathrm{e}} \\
\%\end{array}$ & $\begin{array}{c}\mathrm{CoV}^{\mathrm{f}} \\
\%\end{array}$ & $\begin{array}{c}\text { Mean } \\
\%\end{array}$ & $\begin{array}{l}\mathrm{SD} \\
\%\end{array}$ & $\begin{array}{c}\mathrm{CoV} \\
\%\end{array}$ & \\
\hline - > $2000 \mu \mathrm{m}$ & 0.0 & 0.0 & --- & 0.0 & 0.0 & --- & 0 \\
\hline - $850 \mu \mathrm{m}$ to $2000 \mu \mathrm{m}$ & 69.6 & 3.7 & 5.3 & 15.9 & 8.3 & 52.2 & 0 \\
\hline - $425 \mu \mathrm{m}$ to $850 \mu \mathrm{m}$ & 17.8 & 3.1 & 17.4 & 54.8 & 3.3 & 6.0 & 0 \\
\hline - $180 \mu \mathrm{m}$ to $425 \mu \mathrm{m}$ & 9.3 & 1.6 & 17.2 & 24.0 & 4.3 & 18.0 & 24 \\
\hline - $150 \mu \mathrm{m}$ to $180 \mu \mathrm{m}$ & 1.0 & 0.6 & 56.5 & 2.6 & 0.7 & 27.8 & 13 \\
\hline
\end{tabular}

"Panel 127 has a thick-oil overlayer; MG indicates "manually ground."

b Panel 179 has a thin-latex overlayer; MG indicates "manually ground."

" Panel 279 has a thick-oil overlayer; FG indicates "freezer-mill ground."

¿ Mean of three measurements.

'SD indicates standard deviation.

${ }^{\mathrm{C}} \mathrm{CoV}$ indicates coefficient of variation.

g This is a single measurement and, consequently, no statistics were calculated. Uncertainty is assumed to be of the order of those of Panels 127 and 179.

Panel 279, which had attributes similar to those of Panel 127, was selected for the five-factor experiment. In the previous NIST UE/ASV study [7], the mean lead recovery (10 analyses) for Panel 279 was $39 \%$, with values ranging from $14 \%$ to $67 \%$. Table 7 summarizes the specimenpreparation details for the four groups of specimens designated as low mass/small particle size, low mass/large particle size, high mass/small particle size, and high mass/large particle size. Table 8 gives the results of mass measurements made for the specimens in each of the four groups. The mean masses of the "small mass" and "large mass" specimens were essentially $25 \mathrm{mg}$ and $100 \mathrm{mg}$, respectively. It is noted that, in 1995, Grohse, Luk, Hodson, Wilson, Gutknecht, Harper, Beard, Lim, and Breen [14] recommended that specimens having $\approx 100 \mathrm{mg}$ mass be used for ultrasonic extraction of lead from paint specimens. This value is the same as that of the "high mass" specimens in the current study. It is also noted that the UE/ASV supplier's instructions state that the specimen mass should not be greater than $200 \mathrm{mg}$.

2.3.2 Reference Material Samples. Two reference material samples were included in the UE/ASV analyses: NIST Standard Reference Material (SRM) 1579 and Environmental Lead Proficiency Analytical Testing (ELPAT) Sample 032. The lead content (by mass fraction) of SRM 1579 was $11.87 \%$ [15]; whereas that ${ }^{\dagger}$ of ELPAT 032 was $1.45 \%$. Both samples were finely ground powders. In the case of SRM 1579, essentially all particles pass a $45 \mu \mathrm{m}$-mesh sieve (No. 325) [15]; whereas

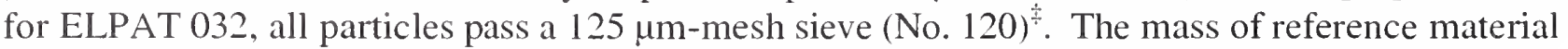
specimens analyzed in the current UE/ASV study was generally between $80 \mathrm{mg}$ and $120 \mathrm{mg}$.

The limitation on specimen mass (i.e., $200 \mathrm{mg}$ ) is only given in the ASV supplier's instructions for analyses for which results are to be reported as percent lead by mass fraction. These instructions indicate that, for results to be reported in $\mathrm{mg} / \mathrm{cm} 2$. the sample collected is to be sonicated.

'Personal communication from Dr. William Gutknecht of the Research Triangle Institute (RTI), who provided the ELPAT sample. Williams et al. [2] include an overview of the RTI procedure used to assign a mean lead level to an ELPAT sample.

Personal communication from Dr. David Binstock of the Research Triangle Institute (RTI). 
Table 7. Preparation details for specimens designated as low or high mass, and small or large particle size

\begin{tabular}{|c|c|c|}
\hline \multirow{2}{*}{$\begin{array}{c}\text { Mass } \\
\text { Designation }\end{array}$} & \multicolumn{2}{|c|}{ Particle Size Designation } \\
\hline & Small & Large \\
\hline Low & $\begin{array}{l}\text { - Randomly sample Panel } 127 \text { ten times } \\
\text { using a No. } 4 \text { cork borer }{ }^{\text {. }} \\
\text { - Place all samplings in a tared freezer- } \\
\text { mill grinding container; weigh total mass } \\
\text { using an analytical balance. } \\
\text { - Grind at liquid nitrogen temperature for } \\
\text { about } 5 \text { min using the freezer mill. } \\
\text { - Measure about } 25 \text { mg of the ground } \\
\text { material into a tared sonicator tube using } \\
\text { a spatula. } \\
\text { - Determine the specimen mass using an } \\
\text { analytical balance. }\end{array}$ & $\begin{array}{l}\text { - Randomly sample } 28 \text { specimens from } \\
\text { Panel } 127 \text { using a No. } 1 \text { cork borer. } \\
\text { - Place each specimen in a tared sonicator } \\
\text { tube; determine the specimen mass using } \\
\text { an analytical balance. } \\
\text { - Manually grind the specimen using a } \\
\text { plastic paint-crushing rod. after cooling } \\
\text { in dry ice. }\end{array}$ \\
\hline High & $\begin{array}{l}\text { - Randomly sample Panel } 127 \text { thirty-six } \\
\text { times using a No. } 4 \text { cork borer. } \\
\text { - Place about one half of the specimens in } \\
\text { a tared freezer-mill grinding container; } \\
\text { weigh total mass using an analytical } \\
\text { balance. } \\
\text { - Grind at liquid nitrogen temperature for } \\
\text { about } 5 \text { min using the freezer mill. } \\
\text { - Measure about } 100 \text { mg of the ground } \\
\text { material into a tared sonicator tube using } \\
\text { a spatula. } \\
\text { - Determine the specimen mass using an } \\
\text { analytical balance. } \\
\text { - Repeat the liquid nitrogen grinding and } \\
\text { specimen weighing on the remaining } \\
\text { material sampled from Panel } 127 \text {. }\end{array}$ & $\begin{array}{l}\text { - Randomly sample } 28 \text { specimens from } \\
\text { Panel } 127 \text { using a No. } 4 \text { cork borer. } \\
\text { - Place each specimen in a tared sonicator } \\
\text { tube; determine the specimen mass using } \\
\text { an analytical balance. } \\
\text { - Manually grind the specimen using a } \\
\text { plastic paint-crushing rod, after cooling } \\
\text { in dry ice. }\end{array}$ \\
\hline
\end{tabular}

${ }^{a}$ A No. 4 cork borer was used for these low-mass specimens to expedite sampling. because the samples obtained were subsequently placed in the freezer mill, ground, and subsampled by mass.

Table 8. Mass measurements as related to specimen mass and particle size

\begin{tabular}{|c|c|c|c|c|}
\hline \multirow[b]{2}{*}{$\begin{array}{c}\text { Mass and Particle Size } \\
\text { Designation }\end{array}$} & \multicolumn{4}{|c|}{ Mass Measurements } \\
\hline & $\frac{\text { Mean }^{\mathrm{a}}}{\mathrm{mg}}$ & $\begin{array}{l}\text { Min } \\
\mathrm{mg}\end{array}$ & $\begin{array}{l}\operatorname{Max} \\
\operatorname{mg}\end{array}$ & $\begin{array}{c}\mathrm{CoV}^{\mathrm{b}} \\
\%\end{array}$ \\
\hline $\begin{array}{l}\text { Low Mass } \\
\text { Large Particle Size }\end{array}$ & 25.2 & 18.8 & 30.6 & 13 \\
\hline $\begin{array}{l}\text { High Mass } \\
\text { Large Particle Size }\end{array}$ & 99.4 & 79.5 & 125.5 & 12 \\
\hline $\begin{array}{l}\text { Low Mass } \\
\text { Small Particle Size }\end{array}$ & 25.4 & 20.2 & 29.8 & 11 \\
\hline $\begin{array}{l}\text { High Mass } \\
\text { Small Particle Size }\end{array}$ & 100.8 & 98.9 & 104.1 & 1.5 \\
\hline
\end{tabular}




\section{RESULTS, ANALYSIS, AND DISCUSSION \\ 3.1 Factors Affecting UE Extraction from Laboratory-Prepared Paint Films}

3.1.1 Preliminary Experiment Including Sonicator Effect. As indicated in the introduction, the limited evidence from the previous NIST UE/ASV study suggested that specimen grinding and sonication may have contributed to the low lead recoveries from the paint-film panels [7]. Additionally, a key experimental variable affecting recovery was the overlayer (Table 1). Paintfilm panels having a thick-oil overlayer had lower lead recoveries than did those with a thin-latex overlayer [7]. Because the mass of specimens having a thin-latex overlayer was, in general, approximately one quarter of that of specimens having a thick-oil overlayer, a question was raised whether specimen mass might be a factor affecting UE extraction. Thus, grinding, sonication, and specimen mass were the primary variables investigated in the preliminary experiment. Observations from this preliminary experiment were used in designing the main experiment investigating factors affecting ultrasonic extraction.

Table 9 lists nine experimental variables included in the preliminary experiment along with an indication as to whether the results suggested that the factor be included in further study. Decisions on whether to include a variable in the main experiment were based on whether lead recovery from Panel 127 was enhanced versus that found in the previous NIST UE/ASV study. Only the results for sonicator power, and sonication temperature and time are discussed herein.

Sonicator power was the focus of the preliminary experiment under the presumption that higher power should result in greater lead recovery. Nine sonicators (Table 3 ) having power ranging from $45 \mathrm{~W}$ to $833 \mathrm{~W}$ were examined. The EPA has recommended that sonicators used for extraction of lead from paint have a minimum power of $53 \mathrm{~W}[10,11]$. Consideration of this recommendation raised the possibility that the $45 \mathrm{~W}$ sonicator (Table 3 ; No. 1), supplied with the UE/ASV apparatus and used in the previous NIST UE/ASV study, was underpowered—contributing to the low lead recoveries from the NIST paint-film panels.

Figure 1 shows plots of lead recovery versus sonicator power for UE/ASV analyses of specimens from Panel 127. SRM 1579, and ELPAT 032. The error bars in this and other figures in the report represent one standard deviation. No error bars are included in Figure 1 for the SRM and ELPAT plots, because the reference materials were analyzed only once with each sonicator. Previously reported UE/ASV analyses of SRM 1579 had coefficients of variation $(\mathrm{CoV})$ of about $\pm 6 \%$ [1].

\footnotetext{
Although it could not be ruled out that the overlayer effect found in the previous NIST UE/ASV study was not associated with the type of overlayer paint, i.e., latex versus oil, an effect due to potential differences in grinding latex and oil paints was considered unlikely. All specimens were ground after cooling in dry ice and both types were therefore assumed to be below their glass transition points during grinding and, thus, comparably brittle. Moreover, Grohse et al. [10] and Luk et al. [11] have indicated that latex paint can be more difficult to grind than oil paint. Thus, if the previously found overlayer effect were associated with paint type, it might be expected that the "more-difficult-togrind" latex specimens might have yielded generally lower lead recoveries than the oil specimens. Nevertheless, an experiment investigating the effect of paint type on lead recovery needs to be conducted.

A basic parameter characterizing sonicators is power density, which is the electrical power into the transducer divided by the transducer radiating surface area [16]. Because the EPA recommendation [10,11] makes power a bath parameter of interest to the lead abatement community, the recovery data are presented as related to power. Moreover, power density values for many of the sonicators used in the study were not readily available. Estimates of power density were approximated by dividing sonicator power by the bath surface area (because the transducers are normally attached to the bottom of the bath). Plots of lead recovery versus estimated power density were similar to those in Figure 1 ; statistical analysis found no significant relationship between recovery and power density for specimens sampled from Panel 127 , SRM 1579, and ELPAT 032.
} 
Table 9. Variables examined in the preliminary experiment investigating factors affecting UE extraction

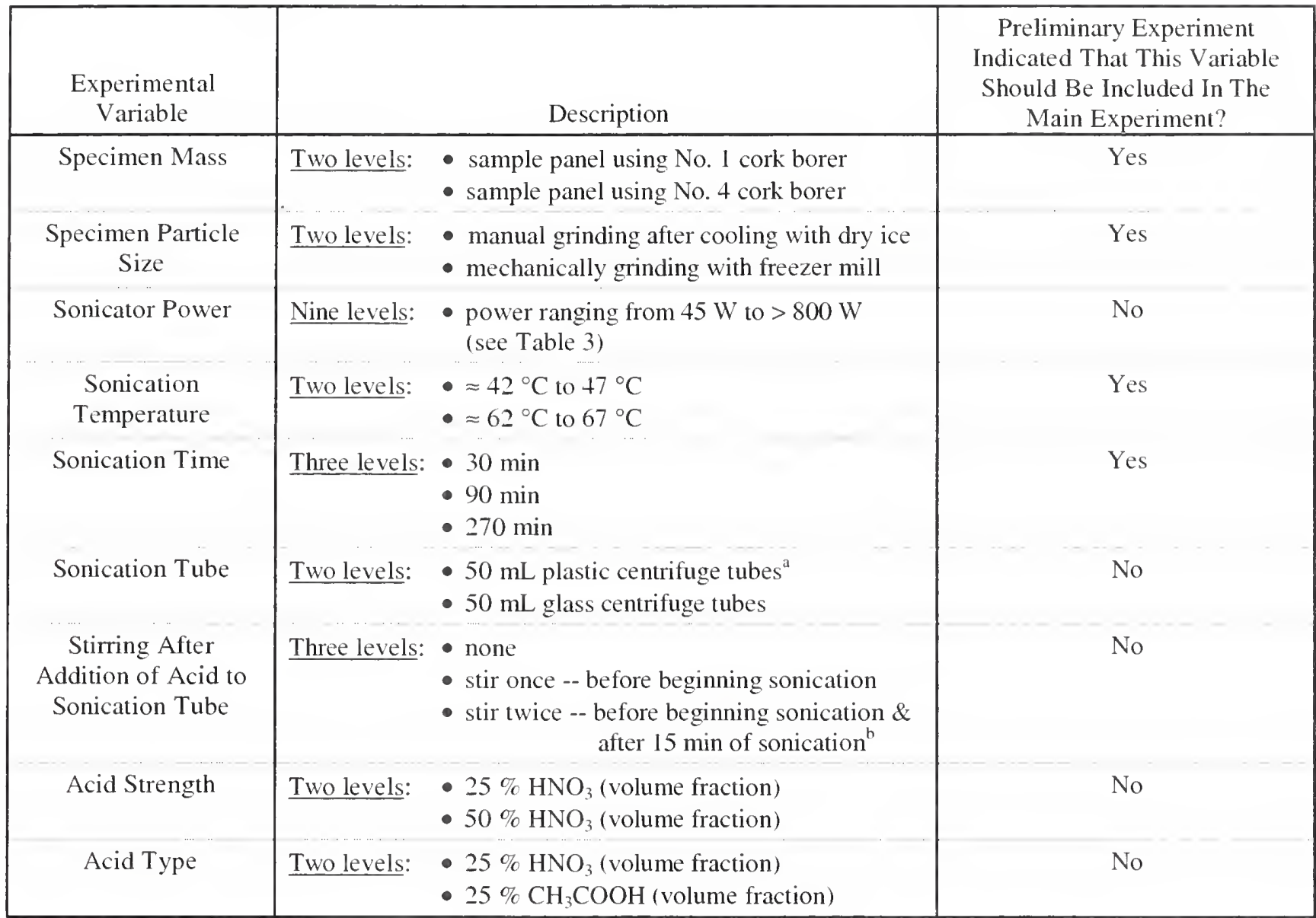

${ }^{a}$ The $50 \mathrm{~mL}$ plastic centrifuge tubes were supplied with the UE/ASV apparatus.

${ }^{\mathrm{b}}$ Sonication was momentarily stopped for stirring.

From Figure 1, it is evident that, for paint film Panel 127, the lead recoveries were low. Statistical analysis indicated no significant relationship between recovery and power ( $p$-value $=0.3715$ ). The range of recovery was from $21 \%$ to $44 \%$, which was almost the same as that found in the previous NIST UE/ASV study in which all measurements were made using Sonicator No. 1. That is, for Panel 127, none of the eight additional sonicators used in the preliminary testing provided statistically-significant, improved lead recovery over that previously obtained using Sonicator No. 1. The lack of a sonicator effect was consistent with the findings of Grohse et al. [14] who reported that lead recoveries for some "real-world" paint specimens were similar when the extractions were performed with either a $53 \mathrm{~W}$ or a $100 \mathrm{~W}$ sonicator.

In contrast to Panel 127, SRM 1579 and ELPAT 032 gave lead recoveries that were greater than $85 \%$ (Figure 1). Statistical analysis of the SRM 1579 and ELPAT 032 data in Figure 1 found no relationship between recovery and sonicator power (p-value $=0.4029$ ). In the case of SRM 1579, the values were similar to the $95 \%$ recovery previously reported by Ashley [1], and the $84 \%$ recovery reported by Williams, Van Hise, and Gutknecht [2]. Recoveries greater than $80 \%$, such as found for SRM 1579 and ELPAT 032, have been designated as being "quantitative" for UE/ASV 
PAINT-FILM PANEL 127
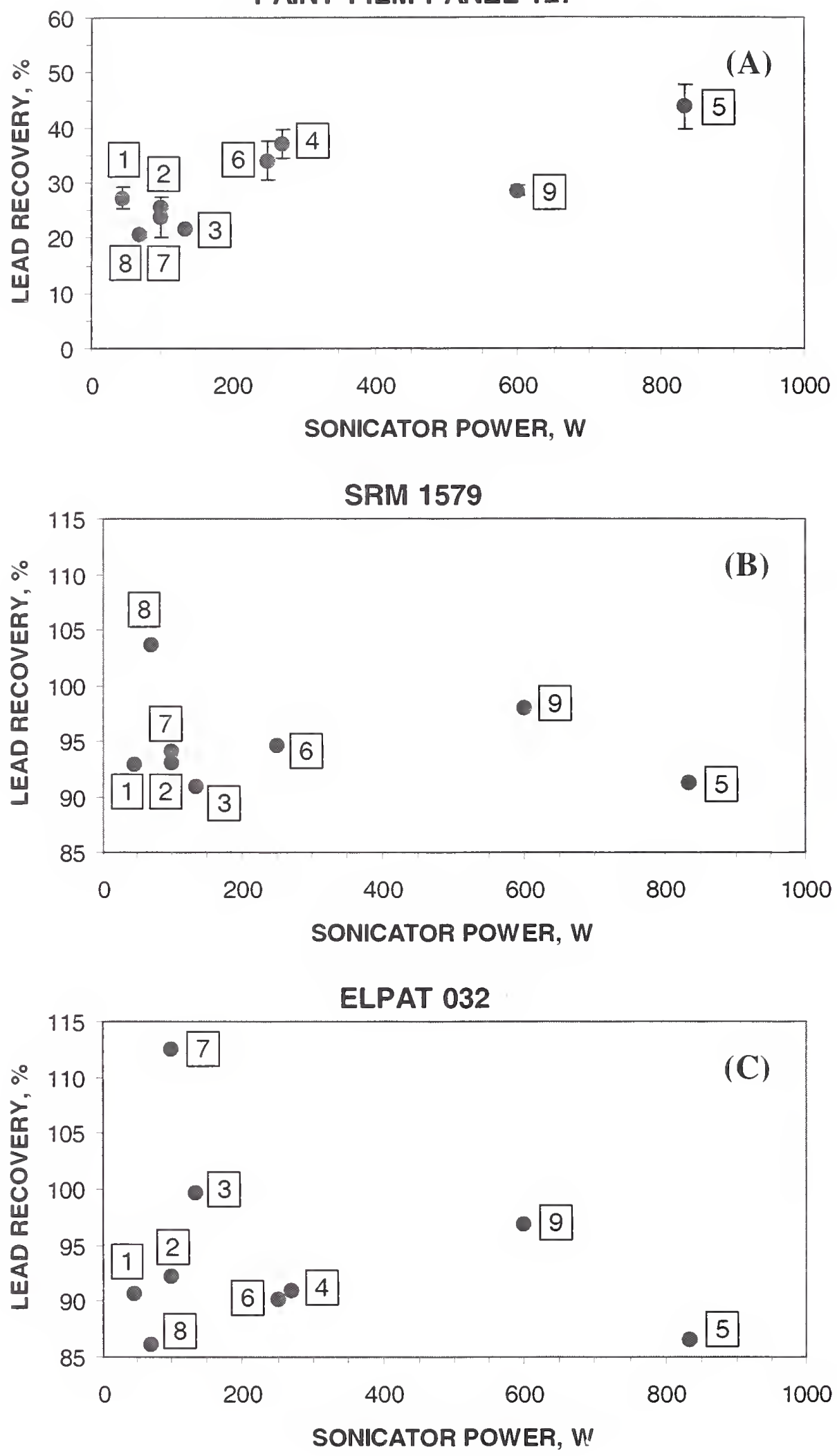

Figure 1. Lead Recovery Versus Sonicator Power. Numbers in boxes represent the Sonicator Nos. listed in Table 3. The error bars in (A) are one standard deviation. Error bars are not included in (B) and (C) because the data points are for one measurement only. 
analyses. For example, Ashley has taken "quantitative recovery" to be $80 \%$ and above $[1,17]$. Consistent with Ashley, the term, "quantitative," is used in this current report for recoveries greater than $80 \%$.

Figure 2 shows results for varying sonication temperature and time for specimens sampled from Panel 127 which, as indicated above, provided low recovery in the previous NIST UE/ASV study [7] and also in the experiment investigating the effect of various sonicators (Figure 1). Observe in Figure 2 that, as the sonication time increased, the lead recovery increased and was quantitative at 270 min for both temperatures. At those times for which recovery was less than quantitative, greater recovery was found at the higher temperature. These data indicate that, given sufficient time, lead in Panel 127 can be quantitatively recovered using Sonicator No. 1 and the specimen preparation conditions and sonicator temperature described in the previous NIST UE/ASV study [7].

3.1.2 Five-Factor Experiment. The preliminary testing indicated that four variables-specimen mass, specimen particle size, sonication temperature and sonication time-should be included in a systematic experiment investigating factors affecting ultrasonic extraction of lead from laboratoryprepared paint films (Table 9). In spite of the findings that sonicator power did not affect lead recovery (Figure 1), a five-factor, two-level full factorial experiment that included sonicator power (Table 2) was designed. Sonicator power was included because of the EPA recommendation $[10,11]$ that a minimum $53 \mathrm{~W}$ sonicator be used for ultrasonic extraction of lead from paint films.

Lead recovery, listed in decreasing order, is given in Table 10 for each of the 32 combinations of experimental variables. It ranged from $47 \%$ to $98 \%$, with 26 of the 32 combinations (i.e., $\approx 80 \%$ )

PAINT-FILM PANEL 127

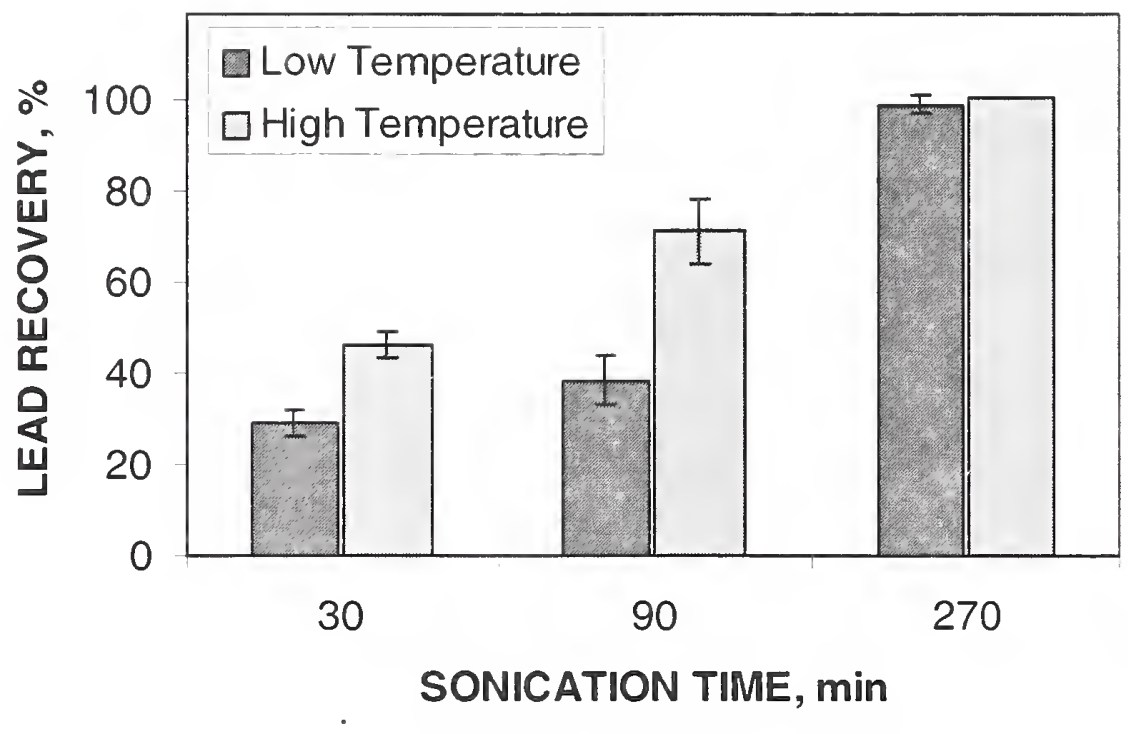

Figure 2. Lead Recovery Versus Sonication Time As Determined at Low and High Temperature for Specimens Sampled from Panel 127. Low and high temperature ranges are $42{ }^{\circ} \mathrm{C}$ to $47{ }^{\circ} \mathrm{C}$, and $62{ }^{\circ} \mathrm{C}$ to $67{ }^{\circ} \mathrm{C}$, respectively. Note that the combined conditions of low temperature and $30 \mathrm{~min}$ time are those that were used in the previous NIST UE/ASV study [7]. The error bars represent one standard deviation. 
Table 10. Lead recovery determined in the five-factor, two-level experiment

\begin{tabular}{|c|c|c|c|c|c|c|c|c|}
\hline \multirow[b]{2}{*}{$\begin{array}{c}\text { Combination } \\
\text { No. }\end{array}$} & \multicolumn{5}{|c|}{ Experimental Variable } & \multicolumn{3}{|c|}{ Lead Recovery } \\
\hline & $\begin{array}{l}\text { Sonicator } \\
\text { Power }\end{array}$ & $\begin{array}{l}\text { Specimen } \\
\text { Mass }\end{array}$ & $\begin{array}{c}\text { Specimen } \\
\text { Particle Size }\end{array}$ & $\begin{array}{c}\text { Sonication } \\
\text { Temperature }\end{array}$ & $\begin{array}{c}\text { Sonication } \\
\text { Time }\end{array}$ & $\begin{array}{c}\text { Mean } \\
\%\end{array}$ & $\begin{array}{l}\text { Spec. }{ }^{a} \\
\text { No. }\end{array}$ & $\begin{array}{c}\mathrm{CoV}^{\mathrm{b}} \\
\%\end{array}$ \\
\hline 1 & High & Small & Small & Low & Long & 97.5 & 3 & 2.1 \\
\hline 2 & High & Small & Small & Low & Short & 96.5 & 3 & 0.7 \\
\hline 3 & Low & Small & Small & High & Short & 96.0 & 4 & 4.0 \\
\hline 4 & Low & Large & Small & Low & Long & 93.3 & 4 & 3.3 \\
\hline 5 & High & Large & Small & Low & Long & 92.8 & 4 & 1.4 \\
\hline 6 & High & Small & Small & High & Long & 92.5 & 4 & 2.3 \\
\hline 7 & Low & Large & Small & High & Long & 92.0 & 3 & 3.9 \\
\hline 8 & Low & Large & Small & High & Short & 91.9 & 3 & 3.1 \\
\hline 9 & High & Large & Large & High & Long & 91.4 & 3 & 5.1 \\
\hline 10 & High & Large & Small & High & Short & 90.9 & 3 & 1.2 \\
\hline 11 & Low & Small & Small & Low & Short & 90.7 & 3 & 10.2 \\
\hline 12 & Low & Small & Small & Low & Long & 90.1 & 3 & 4.2 \\
\hline 13 & High & Large & Small & Low & Short & 88.7 & 4 & 1.2 \\
\hline 14 & Low & Small & Small & High & Long & 88.5 & 4 & 11.5 \\
\hline 15 & High & Large & Small & High & Long & 88.1 & 3 & 4.2 \\
\hline 16 & High & Small & Small & High & Short & 87.9 & 4 & 11.9 \\
\hline 17 & Low & Large & Small & Low & Short & 86.7 & 4 & 4.2 \\
\hline 18 & High & Small & Large & High & Long & 86.1 & 4 & 7.9 \\
\hline 19 & Low & Large & Large & High & Long & 86.0 & 3 & 17.2 \\
\hline 20 & Low & Small & Large & High & Long & 85.3 & 4 & 9.1 \\
\hline 21 & High & Small & Large & High & Short & 81.8 & 3 & 9.6 \\
\hline 22 & High & Large & Large & High & Short & 77.5 & 4 & 6.7 \\
\hline 23 & Low & Small & Large & High & Short & 70.3 & 3 & 10.0 \\
\hline 24 & Low & Large & Large & High & Short & 69.1 & 4 & 10.0 \\
\hline 25 & Low & Large & Large & Low & Long & 69.0 & 4 & 18.0 \\
\hline 26 & High & Large & Large & Low & Long & 68.5 & 4 & 15.0 \\
\hline 27 & High & Small & Large & Low & Long & 62.1 & 3 & 43.7 \\
\hline 28 & Low & Small & Large & Low & Long & 58.6 & 3 & 7.0 \\
\hline 29 & Low & Small & Large & Low & Short & 49.7 & 4 & 20.6 \\
\hline 30 & Low & Large & Large & Low & Short & 47.9 & 3 & 17.2 \\
\hline 31 & High & Small & Large & Low & Short & 47.0 & 4 & 12.3 \\
\hline 32 & High & Large & Large & Low & Short & 46.5 & 3 & 16.9 \\
\hline
\end{tabular}

${ }^{a}$ For one half of the 32 combinations of experimental variables, three replicate determinations of lead recovery were performed; whereas for the other half, four replicates determinations were made. In this manner, 112 specimens were subjected to UE/ASV analysis and, consequently, the sonicator bath was totally full during each use (i.e., 16 sonicator runs times 7 specimens per run $=112$ specimens).

${ }^{\mathrm{b}} \mathrm{CoV}$ indicates coefficient of variation. 
having recoveries greater than the $67 \%$ maximum recovery found for Panel 279 in the previous NIST UE/ASV study [7]. A strong effect due to particle size was observed in Table 10 (statistical analysis follows). For example, note that lead recovery was always quantitative when the particle size was small; whereas it was less than $80 \%$ for 11 of the 16 combinations of experimental variables $(\approx 70 \%$ of the analyses) when the particle size was large.

Figure 3 presents bar plots of lead recovery versus combinations of experimental variables and illustrates the data presented in Table 10. In each plot, the combination of variables for each pair of adjoining bars (i.e., one having a solid fill and the other a striped fill) is the same except for the variable noted in the figure legend. Figures $3 \mathrm{~A}$ and $3 \mathrm{~B}$ imply that sonicator power and specimen mass, respectively, did not have a significant effect on lead recovery; that is, the two adjoining bars for each of the 16 pairs had essentially the same height. In contrast, Figures 3C, 3D, and 3E suggest significant effects due to specimen particle size, sonication temperature, and sonication time, respectively. For example, observe in Figure 3D that, for many of the pairs of adjoining bars, high temperature provided greater recovery than did low temperature.

Analysis of variance of the log of the lead recovery was performed to evaluate the effect of the five factors. The results are summarized in Table 11 using F-statistics and p-values. F-statistics exceeding 1 indicate that an effect is not due to chance. A measure of how unlikely it is that an observed effect is due to chance is provided by the p-value. For purposes of the present report, the p-value is the probability of observing an F-statistic as large or larger than the one obtained, if the effect were not present. Conventionally, effects which have p-values less than 0.05 are considered to be statistically significant. The summary in Table 11 shows that particle size, sonication temperature, and sonication time had a significant effect on the lead recovery. It further shows that the interactions of temperature and particle size and of time and particle size also had significant effects.

Box plots (Figure 4) were used to show the magnitudes of these significant effects. Figure 4A is for particle size, and plots the lead recovery for large and small particles. In this figure, the shaded boxes represent the range of recovery for $50 \%$ of the data points. The white line in each shaded box indicates the median recovery. The "whiskers" (i.e., the brackets above and below the box) represent the smallest and largest points except for the outliers", which are represented by solid lines. In Figure 4A, it is evident that small particles result in significantly higher recovery. The size of the box and spread of the whiskers indicate the influence of the other experimental factors on the recovery. In this case, it shows that the high recovery for small particle size is relatively unaffected by temperature and time.

Figure $4 \mathrm{~B}$ shows the effect of sonication temperature. Here the effect is less dramatic than for particle size. High temperature clearly leads to higher recovery but, in this case, the particle size and time factors dilute the effect of temperature as shown by the fact that the relative sizes of the two boxes are not as different as those in Figure 4A. The effect of sonication time is shown in Figure 4C. Again it is clear that long time leads to higher recovery but, as in the case of temperature, the particle size and temperature factors decrease the effect.

Figures 5A and 5B show the interactions of particle size with temperature and time, respectively. It is apparent that neither an increase in temperature nor time has a great effect on lead recovery for

\footnotetext{
* Outliers, which were identified in developing the box plots (Figure 4), were defined as values that are more than three standard deviations distant from the mean. Such outliers were not excluded from the analysis of variance (Table 11 ).
} 

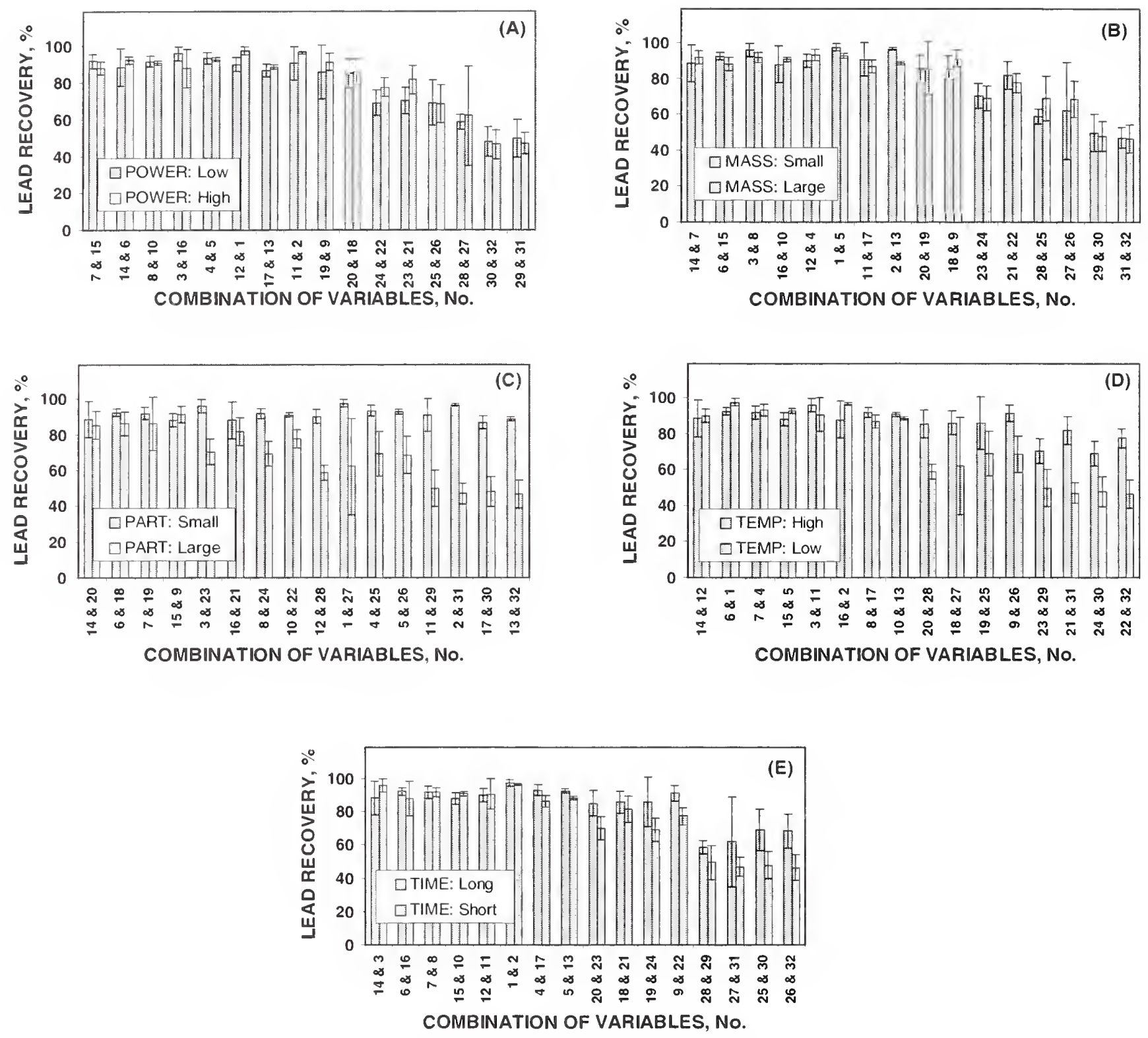

Figure 3. Lead Recovery Versus Combination of Experimental Variables. Figures 3A, 3B, 3C, 3D, and $3 \mathrm{E}$ illustrate the effects of sonicator power, specimen mass, specimen particle size, sonication temperature, and sonication time, respectively. For Figure 3A, low and high sonicator power are $45 \mathrm{~W}$ and $600 \mathrm{~W}$. For Figure 3B, small and large specimen mass are $\approx 25 \mathrm{mg}$ and $\approx 100 \mathrm{mg}$. For Figure $3 \mathrm{C}$, small particles are all $<425 \mu \mathrm{m}$, with the majority $<180 \mu \mathrm{m}$; whereas large particles are for the most part $>425 \mu \mathrm{m}$. For Figure 3D, low and high sonication temperature ranges are $42{ }^{\circ} \mathrm{C}$ to $47^{\circ} \mathrm{C}$, and $62{ }^{\circ} \mathrm{C}$ to $67^{\circ} \mathrm{C}$, respectively. For Figure 3E, short and long sonication times are $30 \mathrm{~min}$ and $90 \mathrm{~min}$, respectively. The error bars represent one standard deviation. For a description of a given combination of experimental variables, the reader is referred to Table 10. 
Table 11. Analysis of variance on the log recovery for the five-factor experiment

\begin{tabular}{|l|c|c|}
\hline & \multicolumn{2}{|c|}{ Analysis Result } \\
\cline { 2 - 3 } Main Effects and Interactions & 173.97 & P-Value \\
\hline Main Effects & 60.12 & 0.0001 \\
Particle size & 23.33 & 0.0001 \\
Temperature & 0.53 & 0.0001 \\
Time & 0.10 & 0.47 \\
Sonicator power & & 0.7536 \\
Specimen mass & 65.85 & \\
Two-Way Interactions & 19.47 & 0.0001 \\
Temperature - Particle size & 3.25 & 0.0001 \\
Time - Particle size & 0.16 & 0.0744 \\
Temperature - Time & 2.48 & 0.6903 \\
Temperature - Specimen mass & 0.26 & 0.1186 \\
Time - Specimen mass & 0.01 & 0.6105 \\
Sonicator - Temperature & 0.00 & 0.9415 \\
Sonicator - Time & & 0.9802 \\
Sonicator - Specimen mass & 0.97 & \\
Three-Way Interactions & & 0.3260 \\
Temperature - Time - Particle size & & \\
\hline
\end{tabular}

small particles. For large particles, however, lead recovery is substantially improved at high temperature and long time.

Mean recoveries for each of the eight combinations of the three significant experimental variables-specimen particle size, sonication temperature, and sonication time-are plotted in Figure 6. Consistent with Figures 4 and 5, particle size has the greatest effect on lead recovery. When the particle size was small, mean lead recovery was quantitative regardless of the conditions of sonication temperature and time. In contrast, when the particle size was large, only the combination of high temperature and long time produced a mean recovery that was quantitative. The lowest mean recovery, $\approx 48 \%$, was for the combination of large particle size, low temperature, and short time, which were the specimen preparation and sonication conditions used in the previous NIST UE/ASV study [7].

The importance of particle size (Figures 4 through 6) provides an explanation for the findings in the previous NIST UE/ASV study [7] that lead levels determined according to the study protocol were often considerably less than the test panel lead levels. In the previous NIST study, the specimens were not ground to a sufficiently small particle size to allow total lead extraction under the temperature and time conditions used for sonication. " The importance of particle size may also explain why in the previous study, three experimental variables-operator, substrate, and overlayer - had significant effects. In the case of the operator, it seems plausible that certain operators ground the specimens more finely than others. In the case of substrate, where steel panels were found to yield higher recovery than plaster panels, it is considered that the procedure for sampling the specimen from the paint-film panel may have come into play (See Table 4, section 2).

\footnotetext{
* Observations of ground specimens in the previous UE/ASV study by NIST research staff showed subjectively that the particle size was similar to that of the "large particles" in the current study.
} 

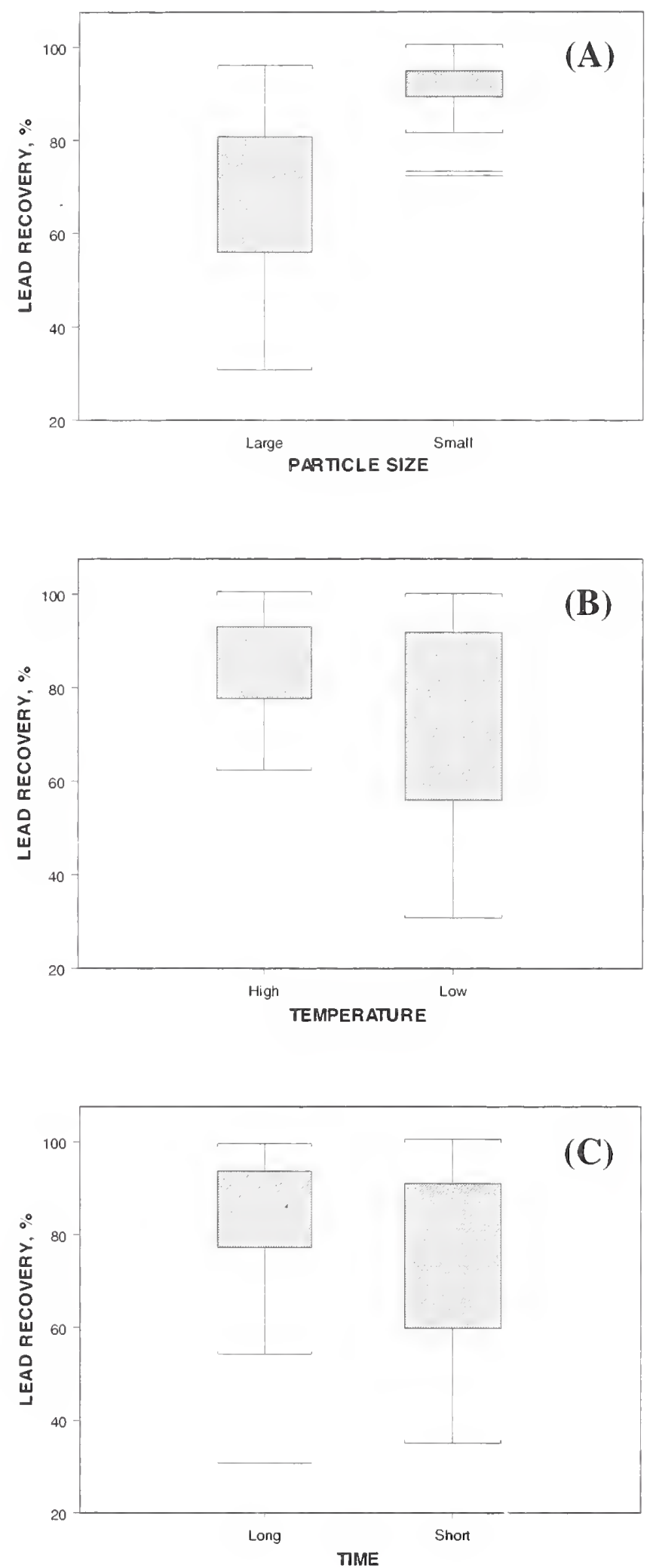

Figure 4. Box Plots of Lead Recovery as a Function of: (A) Particle Size, (B) Sonication Temperature, and (C) Sonication Time. 


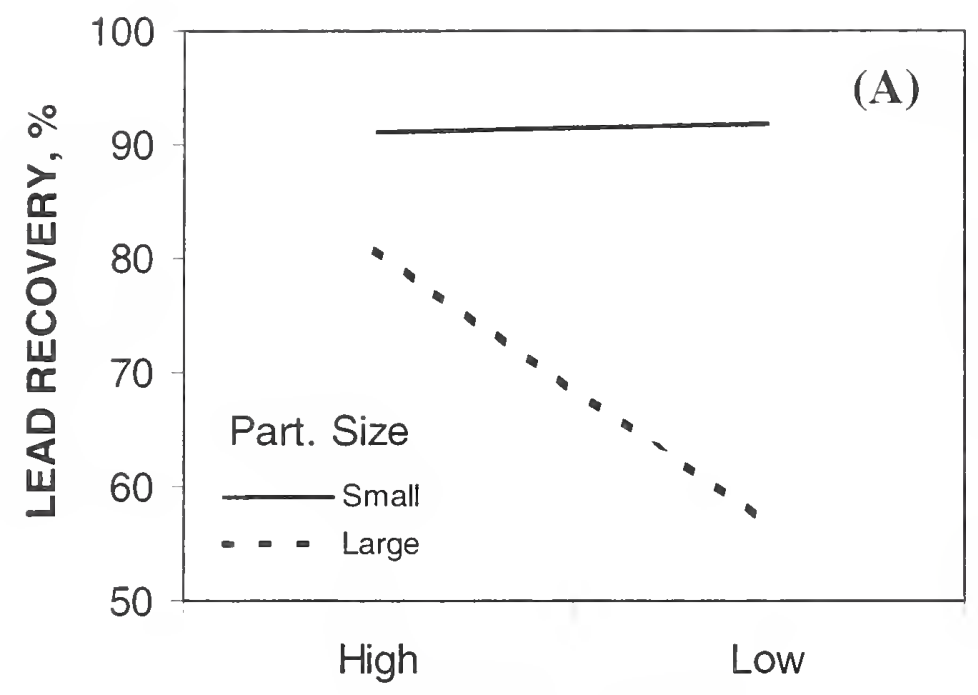

TEMPERATURE

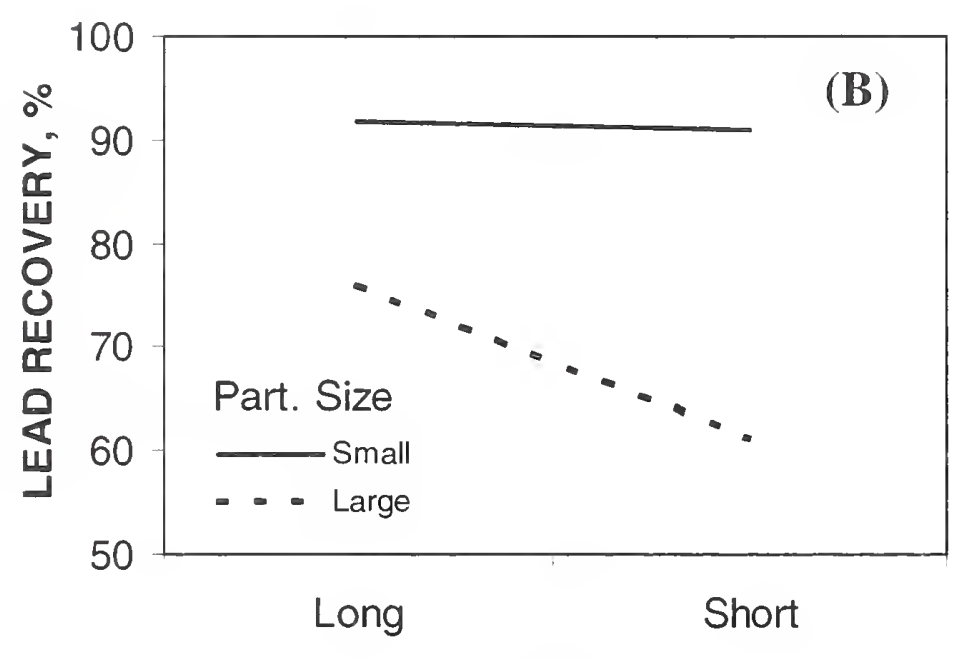

TIME

Figure 5. The Interaction Between Particle Size and (A) Temperature, and (B) Time, Respectively.

When the substrate was plaster, the cork borer normally penetrated through the paint film and into the plaster. A specimen "plug," removed from the cork borer consisted of "a paint-film disk" (and some plaster), which was subsequently ground. Alternatively, when the substrate was steel, the cork borer inscribed a circle in the paint film, which generally remained adhered to the steel substrate. The paint film within the inscribed circle was then scraped from the steel substrate. It is believed that this scraping process for specimens sampled from steel panels generated smaller paintfilm particles than did the process used for grinding the "paint-film disks" taken from the plaster panels. 


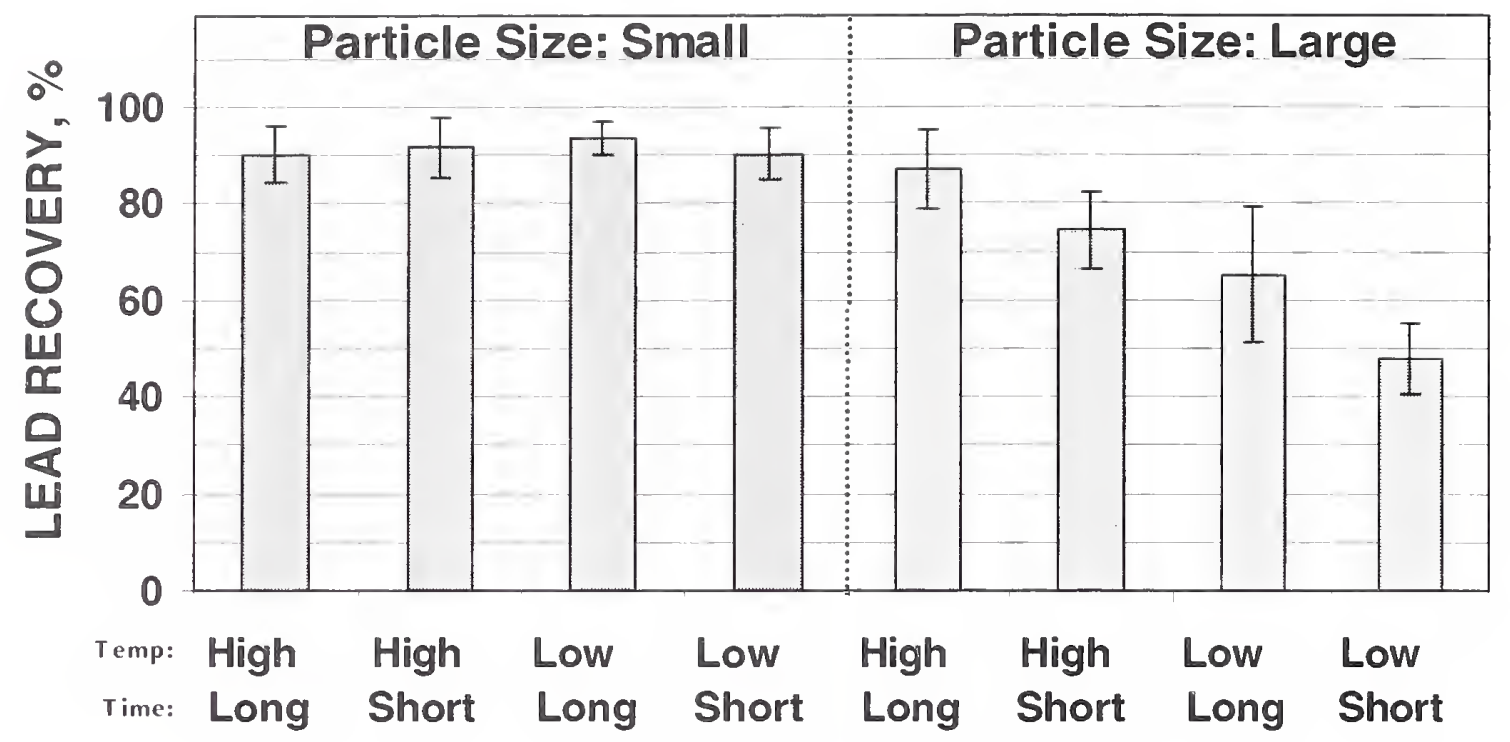

Figure 6. Lead Recovery for the Combinations of Specimen Particle Size, Sonication Time, and Sonication Temperature. Small particles are all $<425 \mu \mathrm{m}$, with the majority $<180 \mu \mathrm{m}$; whereas large particles are for the most part $>425 \mu \mathrm{m}$. Low and high sonication temperature ranges are $42{ }^{\circ} \mathrm{C}$ to $47^{\circ} \mathrm{C}$, and $62{ }^{\circ} \mathrm{C}$ to $67^{\circ} \mathrm{C}$, respectively. Short and long sonication times are $30 \mathrm{~min}$ and $90 \mathrm{~min}$, respectively. The error bars represent one standard deviation.

Concerning the overlayer effect, whereby it was found that paint-film panels having thick-oil overlayers yielded lower lead recoveries than those with thin-latex overlayers, it seems likely that the relative degree of difficulty of grinding the specimens may have played a role. This is consistent with the premise that the more difficult a specimen is to grind, then the more likely it is that the resultant particles are relatively large and, thus, the lead recovery is low. In this regard, it was subjectively found in the current study that specimens having thick-oil overlayers were, for the most part, more difficult to grind than those having thin-latex overlayers. Similarly the operators in the previous NIST UE/ASV study [7] noted that 109 specimens were relatively difficult to grind- $-95 \%$ of these specimens had thick-oil overlayers. Limited data supporting the premise that thick-oil specimens that may be relatively difficult to grind and, consequently, provide larger particle size than thin-latex specimens are given in Table 6. Note that, for the thick-oil Panel 127, $\approx 70 \%$ of the ground specimens had a particle size greater than $850 \mu \mathrm{m}$; whereas for the thin-latex Panel $179, \approx 80 \%$ had a particle size less than $850 \mu \mathrm{m}$. An explanation for why specimens from the thick-oil paint-film panels were generally more difficult to grind than those from thin-latex paintfilm panels was not investigated.

In summary, the results of the five-factor experiment indicated that quantitative recoveries are achievable using the UE/ASV field-portable apparatus provided that the specimens are ground to a sufficiently small particle size, or that extractions are performed at sufficiently high temperature and for long times. The results signifying the importance of particle size are generally consistent with previous findings $[3,10,11,18]$. For example, Hodson, Hardison, Leinbach, Messner, Binstock, and 
Gutknecht [18] reported that finely ground paint chips tend to yield higher lead recoveries than crushed paint chips. Ashley et al. [3] commented that: "The consistency of the grinding process for different types and ages of paints may present problems when one is using this [UE/ASV] method... In cases where paint samples are not easily ground and homogenized (which can be assessed visually), it may be advisable to analyze these samples in the laboratory." Similarly, in the EPA recommendations on a "Standard Operating Procedure" (SOP) for ultrasonic extraction of lead from paint, Luk et al. [10] stated: "Grind until a fine powder is achieved." This SOP directive was slightly modified by Grohse et al. [11] who instructed: "Grind until a particle size equivalent to coarsely ground coffee or cornmeal is achieved." In both EPA reports $[10,11]$, the authors did not provide values of the particle sizes of the "fine powder" or the "coarsely ground coffee or cornmeal." However, in developing the data that supported the SOP, they indicated that the specimens, which included some "real-world" paints and also reference material samples, were mechanically ground to particle sizes $<120 \mu \mathrm{m}$. A particle size of $120 \mu \mathrm{m}$ is comparable to that achieved in the current NIST study using freezer-mill grinding, as approximately two-thirds of the NIST freezer-mill specimens (Table 6) had particles that were less than $150 \mu \mathrm{m}$. Further discussion of grinding and particle size is given in Section 3.4 .

Consistent with the NIST and other results $[3,10,11,18]$ just discussed, the importance of specimen particle size on the recovery of lead, or on that of other metals, ultrasonically extracted from environmental samples other than paint has also been demonstrated. For example, Amoedo, Capelo, Lavilla, and Bendicho [19] found that lead extraction from mussel tissue increased significantly as particle size decreased. Similarly, Nascentes, Korn, and Arruda [20] reported that, for their selected UE conditions, lead recovery from cabbage samples was only quantitative when the particle size was less than $75 \mu \mathrm{m}$. Particle sizes employed in that study ranged from $63 \mu \mathrm{m}$ to $150 \mu \mathrm{m}$.

Although these many authors $[3,10,11,18]$ have discussed the importance of specimen particle size on lead recovery, not all papers are in agreement. Harper and Gutknecht [21] have reported that quantitative lead recoveries have been obtained "by simply coarsely crushing the paint chips" and that "lead recoveries are not dependent on a finely ground sample." It is noted here that the test specimens on which these findings were based were sampled from laboratory-prepared thin film samples without overlayers.

As a final comment, the effect of particle size can explain the differences in the lead recoveries determined for Panel 127 and those for SRM 1579 and ELPAT 032 when extractions were performed using the nine sonicators (Figure 1). Whereas SRM 1579 and ELPAT 032 were fine powders with particle sizes $<125 \mu \mathrm{m}$; the manually ground specimens from Panel $127 \mathrm{had}$ considerably larger particles with $\approx 70 \%$ estimated to range from $850 \mu \mathrm{m}$ to $2000 \mu \mathrm{m}$ (Table 6).

3.1.3 Comparison of ASV Results with ICP Results. Because many lead recoveries in the fivefactor experiment were less than quantitative, it was of interest to examine whether materials extracted from the paint interfered with the ASV electrochemical analysis. Results of a standard addition analysis experiment conducted in the previous NIST UE/ASV study [7] suggested that extracted material from the paint-film panels was not interfering with the ASV measurement. In

\footnotetext{
* The EPA report by Hodson et al. [18] provides some insight regarding particle sizes described as "coarsely ground coffee or cornmeal." Similar to the EPA SOP report [11]. Hodson et al. also recommended that "ground paint should have the consistency of coarsely ground coffee or cornmeal" after manual grinding for $1 \mathrm{~min}$ to $1.5 \mathrm{~min}$. In this case, the authors presented data showing that, after manual grinding, paints taken from a cabinet door and a board had mean particle sizes of $11.7 \mu \mathrm{m}$ and $6.3 \mu \mathrm{m}$, respectively, with maximum values of $198 \mu \mathrm{m}$ and $156 \mu \mathrm{m}$. respectively.
} 
this previous experiment, lead nitrate was added to the extract solutions of specimens sampled from two NIST paint-film panels, and the ASV-measured lead values were comparable to those expected for the spiked solutions. Nevertheless, comparative analysis using ASV and a referee method such as ICP had not been conducted on ultrasonic extracts from any NIST paint-film panel.

Thus, lead concentrations of 24 randomly selected extract solutions from UE/ASV tests conducted in the five-factor experiment on three consecutive days were measured using ICP. The lead recoveries measured in these 24 UE/ASV tests ranged from $31 \%$ to $96 \%$ with a mean of $77 \%$ and varied depending on the combination of five factors incorporated in the given analysis. The ICP measurements were performed in a commercial National Lead Laboratory Accreditation Program (NLLAP) laboratory one day after the ASV measurements were conducted at NIST. Before sending the extract solutions to the NLLAP laboratory, the solutions were filtered through a $0.2 \mu \mathrm{m}$ nylon (Gelman Acrodisc) filter to remove solid residual present after ultrasonic extraction. The extracts were not filtered before performing the ASV analysis. Comparison of the ICP versus ASV analyses is given in Figure 7. The solid line represents the results of a linear regression analysis comparing the ICP data with the ASV data. The dashed lines are the $95 \%$ confidence bounds on the regression. As is evident in Figure 7, the results of the ICP versus ASV data were well correlated $\left(r^{2}=0.988\right)$.

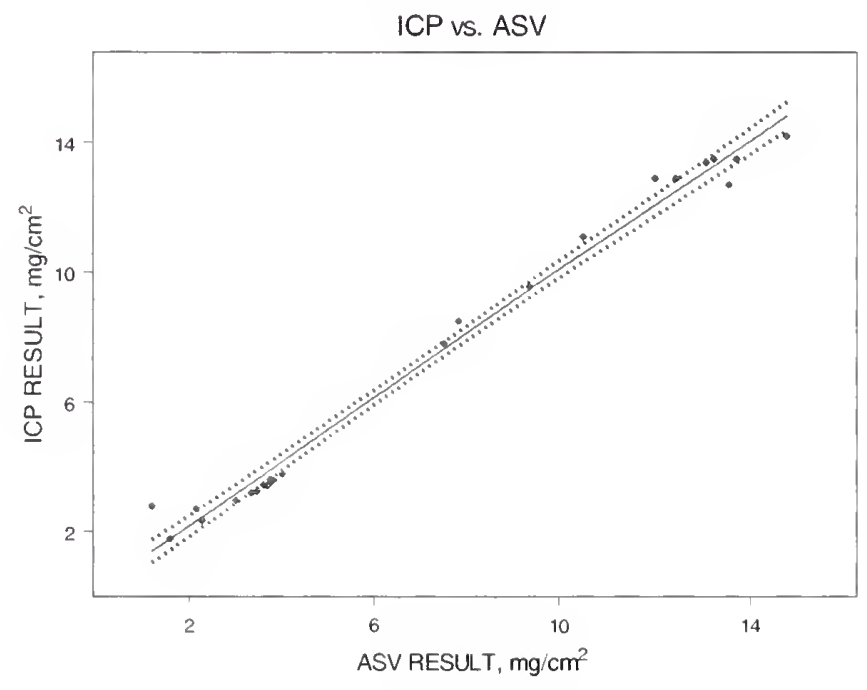

Figure 7. Comparison of the Results of Lead Measurements Performed on Extract Solutions Using ASV and ICP Methods. The solid line represents the results of a linear regression analysis comparison of the ICP data with the ASV data. The dashed lines are the $95 \%$ confidence bounds on the regression. 


\subsection{Lead Extraction from the Series of NIST Paint-Film Panels}

After determining that quantitative lead recovery was achievable for manually ground specimens sampled from Panel 279 when the temperature and time were high and long, respectively, it was of interest to determine whether this combination of extraction conditions would produce quantitative recovery for such specimens from other NIST paint-film panels. The question was: Would quantitative recovery be found for most panels (e.g., $95 \%$ ) when manually ground specimens (i.e., large particle size) were extracted for $90 \mathrm{~min}$ at $\approx 65^{\circ} \mathrm{C}$ ? This is important because, in all likelihood, only manual grinding processes are expected to be included in field protocols for UE/ASV analyses of paint-film specimens. Mechanical grinding in the field using a freezer-mill or similar grinder is a technique that may have practical limitations [18]; for example, liquid nitrogen is needed. If the answer to this question was affirmative, extraction conditions of $90 \mathrm{~min}$ at $\approx 65^{\circ} \mathrm{C}$ might be a practical alternative to the $30 \mathrm{~min}$ and $\approx 45^{\circ} \mathrm{C}$ conditions currently used.

Manually ground specimens sampled from each of the 80 NIST paint-film panels (Tables 5A and $5 \mathrm{~B}$ ) were subjected to sonication at high temperature and long time (i.e., $62{ }^{\circ} \mathrm{C}$ to $67{ }^{\circ} \mathrm{C}$ and $90 \mathrm{~min}$ ). All other specimen preparation and ASV analysis parameters were as stated in Table 4. The results of these UE/ASV analyses are presented in Figure 8A, and are compared with those of the previous NIST UE/ASV study given in Figure 8B. In preparing these figures, lead results recorded as "below the detection limit" were assigned a value of $0.02 \mathrm{mg} / \mathrm{cm}^{2}$, i.e., one-half the detection limit. Tables $12 \mathrm{~A}$ and $12 \mathrm{~B}^{\dagger}$ list the current results in decreasing order of lead recovery for white lead and lead chromate pigments, respectively; these tables also included the results from the previous NIST UE/ASV study [7].

In comparing the current and previous results, it was found that lead recovery was enhanced using high temperature and long time. For example, in Figure 8A, the majority of the recoveries lies close to the complete recovery line and, moreover, were quantitative (i.e., above the $80 \%$ recovery line). In contrast, in Figure $8 \mathrm{~B}$, the majority of the recoveries falls well below the complete recovery line. From Tables 12A and 12B, recovery in the current study was always greater than the mean value determined in the previous NIST UE/ASV study.

Figures 9A, 9B, and 9C plot lead recovery versus panel lead level for overlayer, lead pigment, and substrate types, respectively. Analysis of variance of the log of lead recovery versus panel lead level (Table 13) confirmed that none of the three variables had an effect. For substrate and overlayer, the lack of an effect was in contrast with the findings of the previous NIST UE/ASV study in which these variables showed significant effects [7]. The lack of an effect due to lead pigment type indicates that both white lead and lead chromate pigments (at least in the case of the NIST paint-film panels) are equally extracted by the UE procedure developed for the field portable UE/ASV apparatus.

Although lead recovery was generally enhanced using sonication at high temperature and long time, data in Tables 12A and 12B indicate that these sonication conditions are not adequate for assuring that lead would be quantitatively recovered for all NIST paint-film panels. Approximately $20 \%$ of the analyses—seven white lead specimens and nine lead chromate specimens - had recoveries less than $80 \%$. With exception of Panel 167, these specimens had thick-oil overlayers. Estimates of the

\footnotetext{
"There are 80 and 800 data points represented in Figure 7A (1 operator x 80 paint-film panels), and Figure 7B ( 5 operators $x 80$ paint-film panels x 2 UE/ASV apparatuses), respectively.

${ }^{t}$ Results for panels having a $0 \mathrm{mg} / \mathrm{cm}^{2}$ lead level are not given in these tables. They were found to be "below the detection limit" of the ASV instrument.
} 

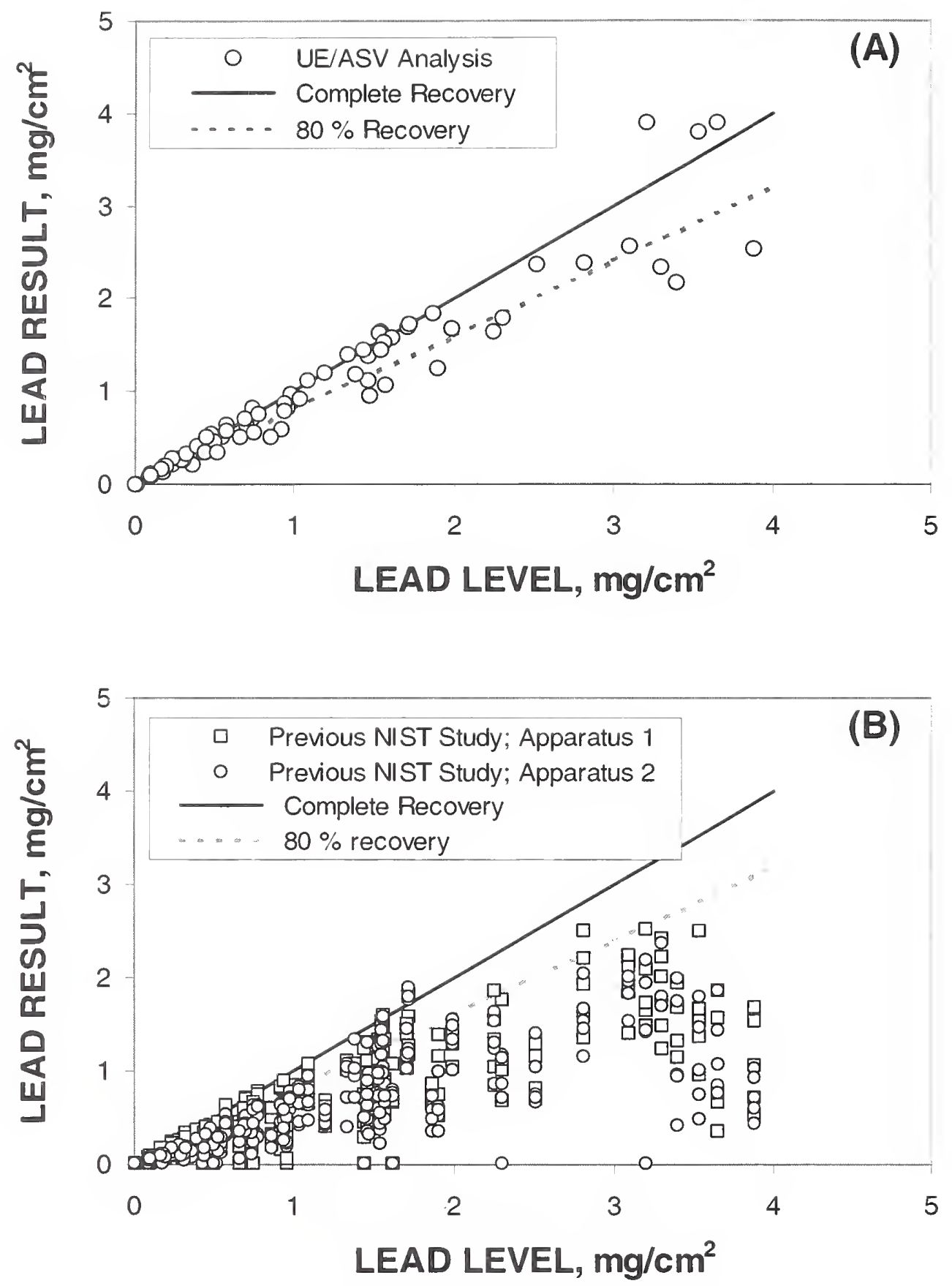

Figure 8. UE/ASV Measured Lead Result Versus Known Panel Lead Level. Plot (A) shows results from the current study in which sonication was performed using high temperature $\left(62{ }^{\circ} \mathrm{C}\right.$ to $67{ }^{\circ} \mathrm{C}$ ) and long time (90 min). Plot (B) gives results from the previous NIST UE/ASV study [7] in which sonication was conducted using low temperature $\left(42{ }^{\circ} \mathrm{C}\right.$ to $47^{\circ} \mathrm{C}$ ) and short time (30 min). All other specimen preparation and sonication conditions were the same. The solid and dashed lines represent complete and $80 \%$ recovery, respectively. 
Table 12A. UE/ASV lead recovery from the NIST paint-film panels determined using high sonication temperature and long sonication time for test panels containing white lead pigment. The results are compared with those of the previous NIST study.

\begin{tabular}{|c|c|c|c|c|c|c|c|c|c|c|}
\hline \multirow{3}{*}{\begin{tabular}{|c|} 
Test \\
Panel \\
1D Code
\end{tabular}} & \multicolumn{4}{|c|}{ Panel Description } & \multicolumn{5}{|c|}{ Lead Recovery-Previous Results ${ }^{a}$} & \multirow{3}{*}{$\frac{\text { Current Results }^{\mathrm{b}}}{\frac{\text { Recovery }}{\%}}$} \\
\hline & \multirow{2}{*}{$\begin{array}{c}\text { Substrate } \\
\text { Type }\end{array}$} & \multicolumn{2}{|c|}{ Overlayer } & \multirow{2}{*}{$\frac{\text { Lead Level }}{\mathrm{mg} / \mathrm{cm}^{2}}$} & \multirow{2}{*}{$\frac{\operatorname{Min}}{\%}$} & \multirow{2}{*}{$\frac{\text { Max }}{\%}$} & \multirow{2}{*}{$\frac{\text { Mean }^{c}}{\%}$} & \multirow{2}{*}{$\frac{S D^{d}}{\%}$} & \multirow{2}{*}{$\frac{\mathrm{CoV}^{\mathrm{e}}}{\%}$} & \\
\hline & & Type & Thick. & & & & & & & \\
\hline 215 & Steel & Latex & Thin & 3.20 & 0.6 & 78.7 & 51.6 & 21.2 & 41 & 121.8 \\
\hline 203 & Steel & Latex & Thin & 0.23 & 49.9 & 107.3 & 80.1 & 20.9 & 26 & 119.2 \\
\hline 188 & Plaster & Latex & Thin & 0.10 & 51.9 & 101.7 & 76.2 & 16.0 & 21 & 116.3 \\
\hline 326 & Steel & Latex & Thin & 0.44 & 44.9 & 92.3 & 70.3 & 14.7 & 21 & 115.0 \\
\hline 224 & Steel & Latex & Thin & 0.58 & 52.8 & 98.3 & 74.8 & 14.6 & 19 & 112.0 \\
\hline 177 & Plaster & Latex & Thin & 3.53 & 14.0 & 71.0 & 38.5 & 16.5 & 43 & 107.7 \\
\hline 251 & Plaster & Latex & Thin & 1.53 & 40.1 & 87.8 & 64.2 & 16.7 & 26 & 105.9 \\
\hline 234 & Plaster & Latex & Thin & 0.19 & 55.0 & 96.6 & 71.0 & 14.4 & 20 & 105.2 \\
\hline 262 & Plaster & Latex & Thin & 0.39 & 50.3 & 98.4 & 74.0 & 14.1 & 19 & 104.6 \\
\hline 222 & Steel & Latex & Thin & 0.32 & 47.8 & 108.5 & 73.9 & 20.1 & 27 & 103.0 \\
\hline 285 & Plaster & Oil & Thick & 0.09 & 21.4 & 104.6 & 61.1 & 24.7 & 40 & 102.5 \\
\hline 237 & Plaster & Oil & Thick & 0.19 & 34.8 & 85.9 & 50.1 & 16.3 & 32 & 100.7 \\
\hline 165 & Plaster & Latex & Thin & 0.28 & 40.3 & 91.9 & 71.3 & 15.0 & 21 & 98.9 \\
\hline 269 & Steel & Latex & Thin & 0.17 & 37.5 & 105.8 & 70.2 & 25.1 & 36 & 98.6 \\
\hline 278 & Steel & Latex & Thin & 0.78 & 38.6 & 100.8 & 75.0 & 20.7 & 28 & 97.0 \\
\hline 226 & Steel & Oil & Thick & 0.54 & 23.6 & 76.6 & 50.0 & 15.2 & 30 & 95.4 \\
\hline 168 & Steel & Oil & Thick & 0.09 & 23.0 & 98.9 & 67.1 & 21.0 & 31 & 94.3 \\
\hline 281 & Plaster & Oil & Thick & 0.50 & 4.0 & 76.7 & 43.2 & 24.1 & 56 & 94.0 \\
\hline 279 & Plaster & Oil & Thick & 1.54 & 14.4 & 66.6 & 39.2 & 16.9 & 43 & 93.9 \\
\hline 135 & Steel & Oil & Thick & 0.23 & 41.0 & 87.8 & 57.1 & 15.1 & 27 & 93.9 \\
\hline 189 & Steel & Oil & Thick & 0.29 & 20.0 & 80.9 & 46.1 & 18.9 & 41 & 91.6 \\
\hline 179 & Plaster & Latex & Thin & 0.70 & 51.8 & 103.5 & 75.5 & 17.6 & 23 & 90.9 \\
\hline 206 & Plaster & Latex & Thin & 0.48 & 32.9 & 88.8 & 64.4 & 19.9 & 31 & 90.4 \\
\hline 256 & Plaster & Latex & Thin & 1.04 & 41.2 & 81.9 & 67.8 & 14.7 & 22 & 87.8 \\
\hline 102 & Plaster & Oil & Thick & 0.96 & 2.1 & 53.6 & 25.5 & 14.7 & 58 & 85.7 \\
\hline 284 & Steel & Latex & Thin & 1.39 & 51.8 & 96.8 & 74.3 & 11.0 & 15 & 85.5 \\
\hline 138 & Plaster & Oil & Thick & 0.31 & 17.7 & 90.4 & 44.0 & 20.1 & 46 & 84.5 \\
\hline 221 & Steel & Latex & Thin & 0.09 & 58.1 & 101.1 & 77.2 & 13.2 & 17 & 83.9 \\
\hline 129 & Plaster & Oil & Thick & 0.43 & 4.7 & 66.0 & 36.1 & 20.3 & 56 & 81.9 \\
\hline 292 & Plaster & Oil & Thick & 0.66 & 3.0 & 69.0 & 39.7 & 21.1 & 53 & 77.1 \\
\hline 123 & Steel & Oil & Thick & 1.46 & 24.3 & 75.8 & 42.8 & 16.1 & 38 & 76.1 \\
\hline 180 & Steel & Oil & Thick & 0.18 & 11.2 & 66.3 & 40.0 & 15.3 & 38 & 70.2 \\
\hline 127 & Plaster & Oil & Thick & 3.88 & 11.5 & 43.7 & 23.9 & 10.8 & 45 & 65.4 \\
\hline 145 & Steel & Oil & Thick & 3.39 & 12.4 & 59.1 & 42.0 & 15.9 & 38 & 64.3 \\
\hline 246 & Steel & Oil & Thick & 0.86 & 20.9 & 70.7 & 37.2 & 16.1 & 43 & 59.0 \\
\hline 130 & Steel & Oil & Thick & 0.36 & 20.6 & 70.0 & 43.3 & 16.3 & 38 & 58.6 \\
\hline
\end{tabular}

The previous NIST results for lead recovery were reported in $\mathrm{mg} / \mathrm{cm}^{2}$ [7]. To allow comparison with the current study, the previous results are presented in this table in "percent recovery." Data points from the previous study that were reported as "below the detection limit" were assigned a value of $0.02 \mathrm{mg} / \mathrm{cm}^{2}$ (i.e., one half the ASV detection limit).

${ }^{\mathrm{b}}$ Each value represents the result of a single measurement; consequently, no uncertainty is given.

"Mean of 10 measurements; the previous study incorporated 5 operators using 2 UE/ASV apparatus.

SD indicates standard deviation.

e $\mathrm{CoV}$ indicates coefficient of variation. 
Table 12B. UE/ASV lead recovery from the NIST paint-film panels determined using high sonication temperature and long sonication time for test panels containing lead chromate pigment. The results are compared with those of the previous NIST study.

\begin{tabular}{|c|c|c|c|c|c|c|c|c|c|c|}
\hline \multirow{3}{*}{$\begin{array}{c}\text { Test } \\
\text { Panel } \\
\text { ID Code }\end{array}$} & \multicolumn{4}{|c|}{ Panel Description } & \multicolumn{5}{|c|}{ Lead Recovery-Previous Results ${ }^{\mathrm{a}}$} & \multirow{3}{*}{$\frac{\text { Current Results }^{b}}{\frac{\text { Recovery }}{\%}}$} \\
\hline & \multirow{2}{*}{$\begin{array}{c}\text { Substrate } \\
\text { Type }\end{array}$} & \multicolumn{2}{|c|}{ Overlayer } & \multirow{2}{*}{$\frac{\text { Lead Level }}{\mathrm{mg} / \mathrm{cm}^{2}}$} & \multirow{2}{*}{$\frac{\mathrm{Min}}{\%}$} & \multirow{2}{*}{$\frac{\operatorname{Max}}{\%}$} & \multirow{2}{*}{$\frac{\text { Mean }^{c}}{\%}$} & \multirow{2}{*}{$\frac{\mathrm{SD}^{\mathrm{d}}}{\%}$} & \multirow{2}{*}{$\frac{\mathrm{CoV}^{\mathrm{e}}}{\%}$} & \\
\hline & & Type & Thick. & & & & & & & \\
\hline 250 & Plaster & Latex & Thin & 0.47 & 48.0 & 91.5 & 69.4 & 16.4 & 24 & 114.6 \\
\hline 216 & Steel & Latex & Thin & 0.74 & 0.0 & 94.7 & 70.4 & 27.8 & 39 & 111.8 \\
\hline 205 & Plaster & Oil & Thick & 3.64 & 9.9 & 51.2 & 30.9 & 14.5 & 47 & 107.1 \\
\hline 187 & Steel & Latex & Thin & 1.55 & 53.5 & 99.4 & 79.9 & 13.6 & 17 & 106.7 \\
\hline 158 & Steel & Latex & Thin & 1.34 & 30.5 & 83.3 & 71.7 & 16.9 & 24 & 104.0 \\
\hline 274 & Plaster & Latex & Thin & 0.69 & 54.5 & 88.2 & 68.5 & 11.4 & 17 & 103.3 \\
\hline 126 & Plaster & Latex & Thin & 1.09 & 43.0 & 98.7 & 71.5 & 16.6 & 23 & 101.9 \\
\hline 140 & Plaster & Oil & Thick & 0.51 & 3.9 & 73.2 & 47.6 & 19.2 & 40 & 101.2 \\
\hline 289 & Steel & Latex & Thin & 0.57 & 58.8 & 111.1 & 79.5 & 15.9 & 20 & 101.2 \\
\hline 329 & Steel & Latex & Thin & 1.72 & 69.2 & 111.1 & 91.0 & 17.2 & 19 & 101.1 \\
\hline 183 & Plaster & Oil & Thick & 1.44 & 1.4 & 86.3 & 37.7 & 25.7 & 68 & 100.9 \\
\hline 178 & Plaster & Oil & Thick & 1.20 & 34.8 & 57.7 & 43.2 & 7.7 & 18 & 100.8 \\
\hline 231 & Steel & Latex & Thin & 0.98 & 59.3 & 87.6 & 73.3 & 7.9 & 11 & 99.4 \\
\hline 249 & Plaster & Latex & Thin & 1.71 & 60.1 & 88.4 & 70.8 & 10.6 & 15 & 99.2 \\
\hline 141 & Plaster & Oil & Thick & 1.86 & 19.8 & 46.9 & 36.9 & 9.0 & 24 & 98.7 \\
\hline 263 & Steel & Latex & Thin & 1.56 & 62.4 & 102.6 & 85.2 & 14.6 & 17 & 97.5 \\
\hline 253 & Plaster & Oil & Thick & 1.62 & 1.2 & 67.5 & 38.9 & 21.1 & 54 & 97.5 \\
\hline 163 & Plaster & Oil & Thick & 0.74 & 16.6 & 66.5 & 39.7 & 13.8 & 35 & 95.6 \\
\hline 148 & Plaster & Latex & Thin & 1.46 & 44.2 & 89.9 & 67.5 & 15.6 & 23 & 94.6 \\
\hline 333 & Plaster & Latex & Thin & 2.51 & 27.0 & 55.9 & 39.3 & 11.4 & 29 & 94.5 \\
\hline 297 & Plaster & Oil & Thick & 0.94 & 27.6 & 65.1 & 45.9 & 14.0 & 31 & 92.0 \\
\hline 142 & Steel & Oil & Thick & 2.81 & 41.2 & 89.3 & 62.4 & 14.9 & 24 & 85.1 \\
\hline 139 & Plaster & Latex & Thin & 1.98 & 51.1 & 78.5 & 66.2 & 8.8 & 13 & 84.2 \\
\hline 323 & Plaster & Latex & Thin & 0.94 & 48.3 & 95.7 & 63.8 & 15.6 & 24 & 84.1 \\
\hline 199 & Steel & Latex & Thin & 3.09 & 45.5 & 72.6 & 61.4 & 8.3 & 14 & 82.8 \\
\hline 308 & Steel & Oil & Thick & 0.43 & 24.5 & 78.6 & 54.7 & 16.7 & 30 & 81.4 \\
\hline 223 & Steel & Latex & Thin & 0.49 & 43.2 & 79.7 & 62.8 & 11.1 & 18 & 81.3 \\
\hline 330 & Plaster & Oil & Thick & 2.30 & 0.9 & 77.3 & 42.2 & 19.7 & 47 & 78.3 \\
\hline 302 & Steel & Oil & Thick & 0.75 & 32.1 & 91.1 & 57.2 & 17.2 & 30 & 75.4 \\
\hline 331 & Steel & Oil & Thick & 2.25 & 38.3 & 83.6 & 59.2 & 15.0 & 25 & 73.2 \\
\hline 167 & Plaster & Latex & Thin & 3.29 & 37.9 & 73.6 & 57.2 & 11.5 & 20 & 70.9 \\
\hline 315 & Steel & Oil & Thick & 1.57 & 30.8 & 85.5 & 50.7 & 15.4 & 30 & 68.0 \\
\hline 310 & Steel & Oil & Thick & 0.52 & 28.3 & 87.3 & 60.3 & 16.1 & 27 & 67.9 \\
\hline 294 & Steel & Oil & Thick & 1.89 & 19.0 & 73.7 & 39.4 & 17.6 & 45 & 66.2 \\
\hline 124 & Steel & Oil & Thick & 1.48 & 21.7 & 86.2 & 50.8 & 18.9 & 37 & 65.0 \\
\hline 113 & Steel & Oil & Thick & 0.92 & 17.6 & 90.9 & 56.1 & 23.7 & 42 & 64.0 \\
\hline
\end{tabular}

${ }^{d}$ The previous N1ST results for lead recovery were reported in $\mathrm{mg} / \mathrm{cm}^{2}$ [7]. To allow comparison with the current study, the previous results are presented in this table in "percent recovery." Data points from the previous study that were reported as "below the detection limit" were assigned a value of $0.02 \mathrm{mg} / \mathrm{cm}^{2}$ (i.e., one half the ASV detection limit).

${ }^{\mathrm{b}}$ Each value represents the result of a single measurement; consequently, no uncertainty is given.

c Mean of 10 measurements; the previous study incorporated 5 operators using 2 UE/ASV apparatus.

${ }^{d}$ SD indicates standard deviation.

¿ $\mathrm{CoV}$ indicates coefficient of variation. 

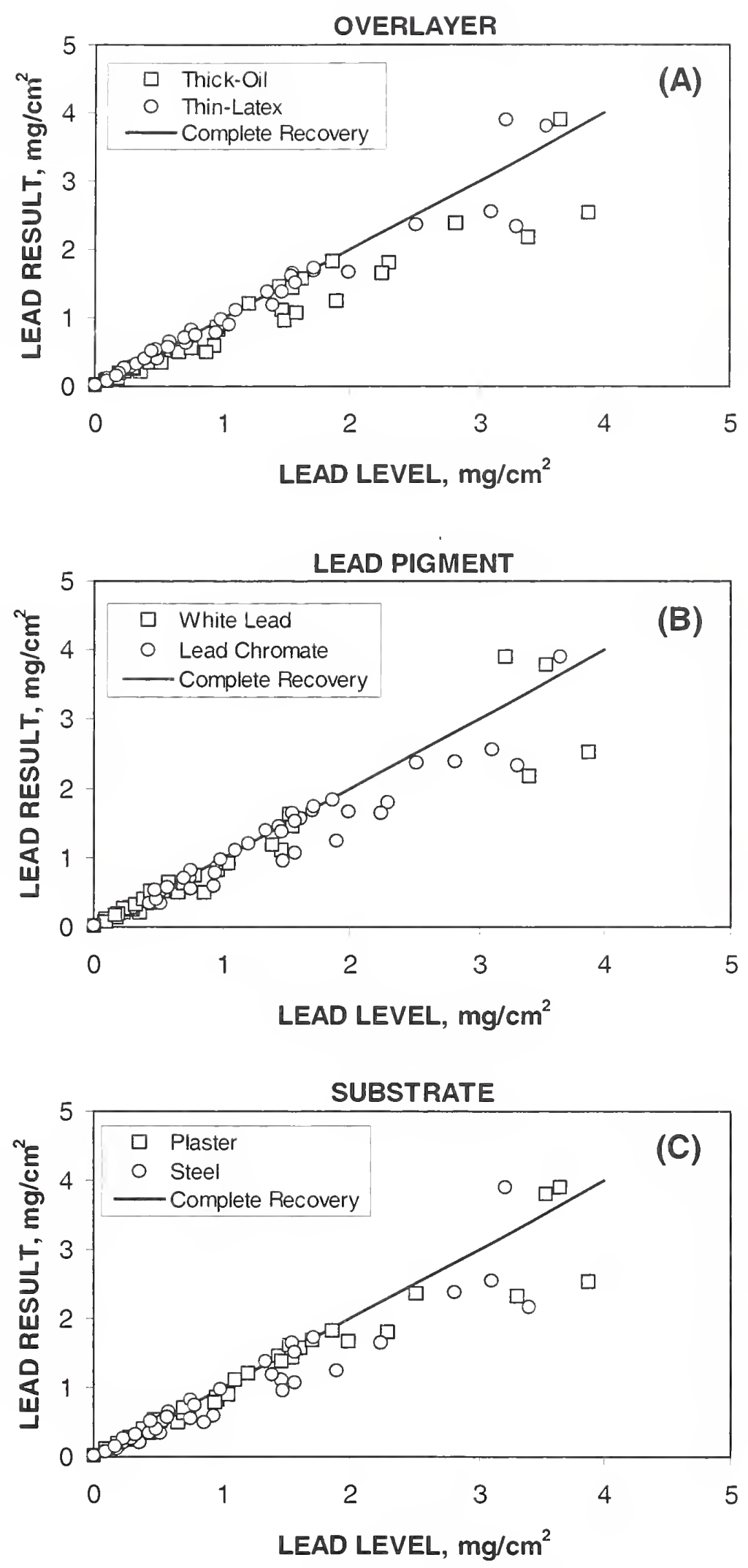

Figure 9. Parametric Effects on Lead Recovery. Plots (A), (B), and (C) illustrate the effect of overlayer, lead pigment, and substrate, respectively. Extractions were performed using high temperature $\left(62{ }^{\circ} \mathrm{C}\right.$ to $\left.67^{\circ} \mathrm{C}\right)$ and long time $(90 \mathrm{~min})$. 
Table 13. Analysis of variance on the log lead recovery for the ultrasonic extraction from the series of 80 NIST paint-film panels

\begin{tabular}{|l|c|c|}
\hline & \multicolumn{2}{|c|}{ Analysis Result } \\
\cline { 2 - 3 } Panel Variable & F-Statistic & P-Value \\
\hline Overlayer Type & 0.09 & 0.7673 \\
Lead Pigment Type & 0.01 & 0.9367 \\
Substrate Type & 0.13 & 0.7168 \\
\hline
\end{tabular}

particle size of the manually ground specimens were not obtained in this experiment. Based on results of the five-factor experiment indicating the importance of particle size (Section 3.1.2) and the observations in the NIST UE/ASV studies that the thick-oil specimens are relatively difficult to grind manually, it seems reasonable to hypothesize that, in general, the particle size of the 16 specimens for which the lead recovery was less than quantitative was not small enough. This finding suggests that a process is needed to ensure the generation of small particles from the paintfilm specimen before performing an ASV analysis. However, because the use of mechanical grinding, which has been shown to achieve small particles, has been considered impractical for field use [18], a recommendation is made that any protocol for preparing a paint-film specimen for UE/ASV analysis should include an estimation of the resultant particle size of the manually-ground specimens before performing the lead extraction (see Section 3.4).

\subsection{Role of Sonication}

3.3.1 Sonication Characterization. The role of sonication in lead extraction from paint-film specimens was of interest because of the finding that sonicator power had no effect on lead recovery. Many research papers $(1-3,10,11)$ discussing the use of ultrasonic extraction of lead from paint generally do not address the extraction mechanism(s). One consideration has been that sonication provides for "agitation" of the specimen in the extraction solution [14]. Another supposition is that the combination of temperature, pressure, and physical effects associated with ultrasonic cavitation breaks up the solid matrix containing undissolved lead particles, with the concomitant acid-assisted dissolution of the lead [4]. Cavitation is the production of microbubbles in a liquid upon application of a large negative pressure $[22,23]$. The subsequent collapse of these microbubbles is accompanied by a release of intense local energy ${ }^{*}$. For cavitational action to assist lead extraction, the ultrasonic energy must be transmitted through the walls of the sonication tube and cause cavitation within the tube [16,23]. Limited experimentation was conducted to examine whether cavitation occurs in a sonication tube during an ultrasonic extraction conducted according to the protocol (Table 4) used in the UE/ASV analysis of paint-film specimens.

As described by Mason [23], water can be fragmented during cavitation into $\mathrm{H} \bullet$ and $\mathrm{HO} \cdot$ radicals that undergo a series of reactions generating, among other products, hydrogen peroxide $\left(\mathrm{H}_{2} \mathrm{O}_{2}\right)$. The hydrogen peroxide oxidizes iodide ion $\left(\mathrm{T}^{\top}\right)$ to iodine $\left(\mathrm{I}_{2}\right)$ that, in the presence of starch, forms a deep blue color. Mason provides a procedure for this test as follows:

"Place $5 \%$ aqueous $\mathrm{KI}$ solution $(100 \mathrm{~mL})$ in a $250 \mathrm{~mL}$ conical flask and add a few drops of $\mathrm{CCl}_{4}$ followed by a soluble starch indicator $(2 \mathrm{~mL})^{\dagger}$. The flask is then immersed in the

\footnotetext{
For readings on the theory and practical applications of cavitation, see Reference Nos. 16 and 22 through 25.

${ }^{\dagger} \mathrm{KI}$ and $\mathrm{CCl}_{+}$are potassium iodide and carbon tetrachloride, respectively.
} 
ultrasonic bath to a depth where the clear solution suffers maximum disturbance. Within $1 \mathrm{~min}$, the blue starch/iodine color will start to appear and this will deepen as the reaction proceeds."

In the current study, this qualitative starch/iodine test was performed using reagents placed directly in the bath of Sonicator No. 1 and into plastic and glass centrifuge tubes placed in the bath, although the $\mathrm{CCl}_{4}$ was not added until the sonicator was run for $3 \mathrm{~min}$. The amounts of reagents were adjusted appropriately to the sizes of the bath and centrifuge tubes. For both the plastic and glass centrifuge-tube tests, seven tubes (i.e., the maximum accommodated by Sonicator No. 1) were examined simultaneously. The starch/iodine reaction is more sensitive the lower the temperature, and a starch/iodine solution loses color when heated above room temperature, but it returns when the solution cools [26]. In conducting the starch/iodine test in centrifuge tubes, the sonicator bath temperature was $\approx 40^{\circ} \mathrm{C}$ to $45^{\circ} \mathrm{C}$ to be representative of that used in a typical UE/ASV analysis. Color examinations were made periodically during sonication, and after the solutions had cooled to room temperature, $\approx 22{ }^{\circ} \mathrm{C}$. The starch/iodine test conducted directly in the sonicator bath was performed at room temperature.

The results of the starch/iodine tests varied depending on whether the reagents were in the sonicator bath or in the tubes. In the case of the bath, a dark deep blue color was observed within $\approx 40 \mathrm{~s}$ after addition of the $\mathrm{CCl}_{4}$. The initial blue was so deep that deepening with time could not be sensed. This observation supported the premise that cavitation was occurring in the bath-a finding consistent with the results of the aluminum foil performance checks conducted on the sonicator baths as described in Section 2.1.2. In contrast, only slight color change was observed after addition of the $\mathrm{CCl}_{4}$ in the heated centrifuge tubes, and it was not seen in all tubes even after 30 min sonication. The tube in the center of the bath appeared to show the greatest color change. For the plastic tubes, the color was not dark blue, but slightly purple and it appeared to lighten, in some cases, to a yellow hue over time. For the glass tubes, the color was characterized as a slight blue that was faint in comparison to the color intensity observed in the sonicator bath. The centrifuge tubes were cooled over night to room temperature upon which they were re-examined. For both plastic and glass, a small amount of blue precipitate was seen in each of the seven tubes; the solutions were clear to slightly yellow. The interpretation of the starch/iodine test results for the centrifuge tubes is that some cavitation may have taken place in the tubes, but the degree of cavitation was apparently less than that which occurred in the sonicator bath.

In further examination of the question of cavitational action in the tubes, small squares of household aluminum foil ( $15 \mathrm{~mm}$ by $15 \mathrm{~mm}$ by $0.03 \mathrm{~mm}$ ) were placed in each of seven plastic centrifuge tubes along with $5 \mathrm{~mL}$ of soap-containing water. The foil pieces were sonicated in Sonicator No. 1 at a bath temperature at $42{ }^{\circ} \mathrm{C}$ to $47^{\circ} \mathrm{C}$, and examined for signs of perforations or other deterioration. After 1 min sonication, none of the foil pieces appeared damaged. In contrast, recall fiom the aluminum foil check on sonicator performance (Section 2.1.2) that perforations were readily produced in the sonicator bath essentially when the sonicator was turned on. After 30 min with the foil in the sonication tubes placed, only the foil placed in the center tube of the sonicator bath showed damage. In this case, the foil section had broken down ${ }^{*}$ to a powder.

Perhaps with the exception of the center location of the sonicator, the results of the starch/iodine experiment and aluminum foil test appear to be more consistent with a mechanism in which sonication provides for "agitation" of the extraction solution than with a mechanism in which

${ }^{*}$ For comparison, a $15 \mathrm{~mm}$ by $15 \mathrm{~mm}$ piece of aluminum foil was stirred in the water bath for $30 \mathrm{~min}$ at $42{ }^{\circ} \mathrm{C}$ to $47^{\circ} \mathrm{C}$. In this case, no deterioration of the foil was observed. 
cavitational action is contributing, to any notable extent, to extraction. This leads to the supposition that lead extraction from the NIST laboratory-prepared paint-film panels is primarily associated with a diffusion-controlled mechanism. This supposition is supported by the findings that lead extraction efficiency is enhanced by large surface area (i.e., small particles), increased temperature, and prolonged time, [27] and, as is discussed in Section 3.3.2, is the same with or without ultrasound.

Another sonicator parameter of interest is the temperature within each of the centrifuge (i.e., sonicator) tubes during operation, because it can be higher than that of the bath due to ultrasonic heating [23]. Thus, seven plastic tubes containing $5 \mathrm{~mL}$ of distilled water were sonicated in Sonicator No. 1 for $30 \mathrm{~min}$ at $42{ }^{\circ} \mathrm{C}$ to $47^{\circ} \mathrm{C}$. After sonication, the temperature in each tube was recorded using a thermocouple. Duplicate runs were conducted. In Figure 10, the seven circles illustrate (not to scale) the relative locations of the seven sonicator tubes, as placed in the sonicator bath, while the numbers in each circle are the highest temperatures recorded for each run. The temperatures in the seven tubes were not uniform. A maximum temperature difference of $14^{\circ} \mathrm{C}$ was found between some adjacent tubes with the center location at $60^{\circ} \mathrm{C}$ and the two bottom-row tubes at $46{ }^{\circ} \mathrm{C}$. Since the maximum temperature of the bath was $\approx 46{ }^{\circ} \mathrm{C}$, the low temperatures imply that ultrasonic heating of these bottom-row tubes did not occur.

A limited experiment was conducted on whether the lack of temperature uniformity among the seven tubes placed in Sonicator No. 1 might have an effect of lead recovery. The specimens were sampled from Panel 102, which had a white lead pigment, thick-oil overlayer, and plaster substrate.

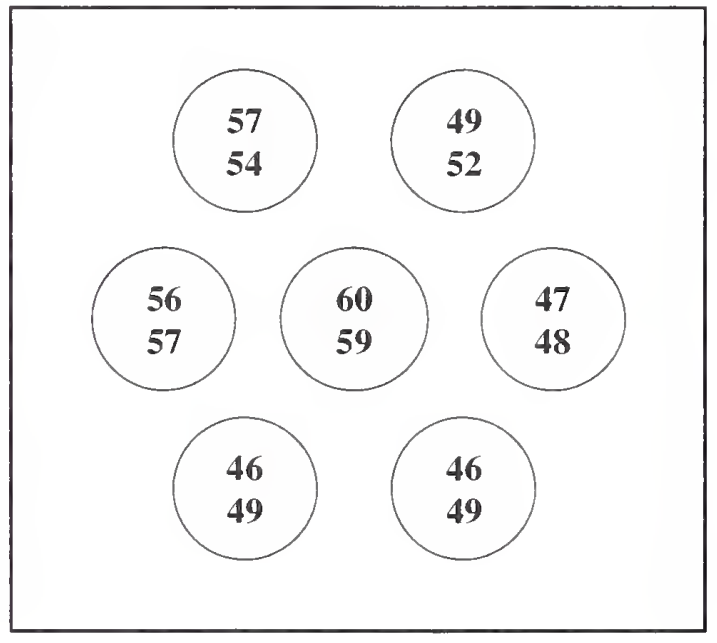

Figure 10. Maximum Temperatures $\left({ }^{\circ} \mathrm{C}\right)$ in Each of the Seven Sonicator Tubes Placed in Sonicator No. 1. The circles represent the relative locations (not to scale) of the tubes. Two runs were conducted, and the two values in each circle are the maximum temperatures recorded for each run after sonication was terminated. The temperature of the sonicator bath was $\approx 42{ }^{\circ} \mathrm{C}$ to $47^{\circ} \mathrm{C}$. 
In the previous NIST UE/ASV study [7], the mean lead recovery (10 measurements) was $25 \%$ with a range from "below the instrument detection limit" to $54 \%$. In the current study, two runs were performed. For each run, seven specimens were manually ground and randomly assigned to the seven tube locations of Sonicator No. 1 for extraction according to the NIST protocol (Table 4). The mean lead recoveries for the two runs as a function of the average tube temperature are presented in Figure 11. No relationship between lead recovery and temperature was observed. It may have been that differences in specimen particle size due to manual grinding overwhelmed any temperature effect.

3.3.2 Lead Extraction Without Ultrasound. In investigating the role of sonication, lead extractions of specimens from selected NIST paint-film panels (Table 14), SRM 1579, and ELPAT 032 were performed without ultrasound using a water bath (for temperature control) with mechanical stirring of the specimen in $25 \% \mathrm{HNO}_{3}$. All specimens were prepared, extracted under varying conditions of temperature and time, and analyzed for lead according to the NIST UE/ASV protocol (Table 4). The results of these extractions were compared to those obtained using Sonicator No. 1.

The results of this experiment (Table 15) indicated that, under the same conditions of temperature and time, comparable lead recoveries were determined with and without ultrasound. In the case of the finely ground specimens from Panel 279 and from SRM 1579 and ELPAT 032, quantitative lead recovery occurred with and without ultrasound at short extraction times regardless of whether the temperature was high or low. In the case of the manually ground specimens, lead recovery using extraction without ultrasound varied with panel and extraction conditions. Using extraction without ultrasound at low temperature and short time, lead recovery for the manually ground specimens was within the range of values measured in the previous NIST UE/ASV study using Sonicator No. 1 at

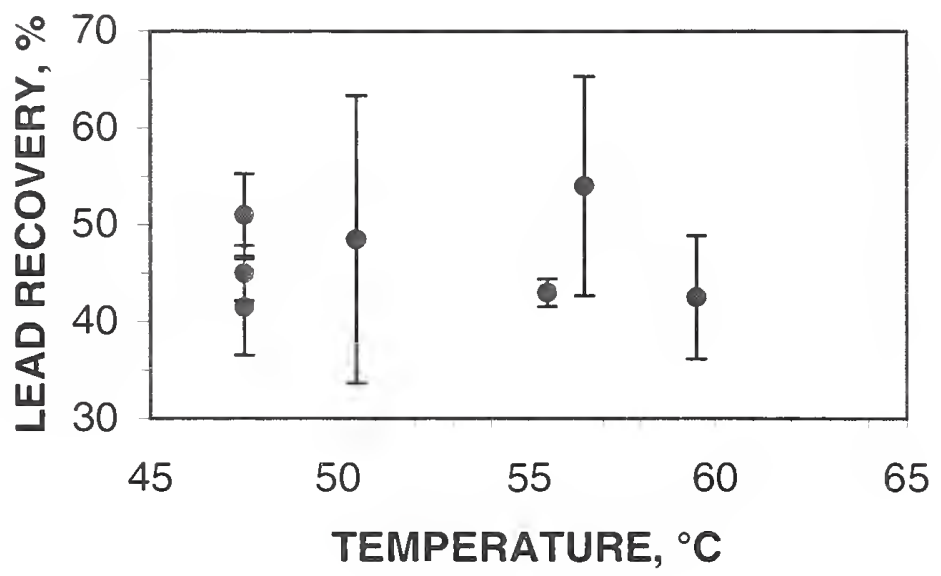

Figure 11. Lead Recovery from Specimens Sampled from Panel 102 Versus Sonication Tube Temperature. The error bars represent one standard deviation. 
Table 14. Paint-film panels for the experiment extracting lead without ultrasound using a water bath with mechanical stirring of the specimen in the acid solution

\begin{tabular}{|c|cccc|c|}
\hline \multirow{2}{*}{$\begin{array}{c}\text { Paint-Film } \\
\text { Panel } \\
\text { ID Code }\end{array}$} & \multicolumn{4}{|c|}{ Panel Description } & \\
\cline { 2 - 4 } & Lead Pigment & Substrate & \multicolumn{2}{c|}{ Overlayer } & \\
\cline { 3 - 4 } & Type & Type & Type & Thickness & Grinding Method \\
\hline 126 & Lead Chromate & Plaster & Latex & Thin & Manual after Cooling in Dry Ice \\
141 & White Lead & Plaster & Oil & Thick & \\
237 & Lead Chromate & Plaster & Oil & Thick & \\
262 & White Lead & Plaster & Oil & Thick & \\
297 & White Lead & Plaster & Latex & Thin & \\
279 & Lead Chromate & Plaster & Oil & Thick & \\
& White Lead & Plaster & Oil & Thick & Mechanical in a Freezer Mill for \\
& & & & & $\approx 5$ min at Liq. N2 Temperatures \\
\hline
\end{tabular}

Table 15. Comparison of lead recovery for extractions performed with and without ultrasound

\begin{tabular}{|c|c|c|c|c|c|c|c|c|}
\hline \multirow{5}{*}{$\begin{array}{c}\text { Sample } \\
\text { Designation }\end{array}$} & \multicolumn{5}{|c|}{$\begin{array}{l}\text { Lead Recovery } \\
\text { Extraction Without Ultrasound } \\
\end{array}$} & \multirow{3}{*}{\multicolumn{3}{|c|}{$\begin{array}{c}\text { Lead Recovery } \\
\text { Extraction With Ultrasound } \\
\text { Extraction Conditions: } \\
\text { Low/Short } \\
\end{array}$}} \\
\hline & \multicolumn{5}{|c|}{ Extraction Conditions: Temperature and Time ${ }^{\mathrm{d}}$} & & & \\
\hline & \multirow{2}{*}{$\frac{\text { High/Long }}{\text { Rep. } 1^{\mathrm{e}}}$} & \multicolumn{2}{|c|}{ High/Short } & \multicolumn{2}{|c|}{ Low/Short } & & & \\
\hline & & Rep. 1 & Rep. 1 & Rep. 1 & Rep. 2 & Mean $^{\mathrm{f}}$ & Min & $\operatorname{Max}$ \\
\hline & $\%$ & $\%$ & $\%$ & $\%$ & $\%$ & $\%$ & $\%$ & $\%$ \\
\hline Panel $127(\mathrm{MG})^{g}$ & 59.4 & 37.4 & 38.5 & 22.7 & 20.9 & 23.9 & 11.5 & 43.7 \\
\hline Panel 141 (MG) & 97.4 & 76.1 & 48.9 & 30.4 & 42.5 & 36.9 & 19.8 & 46.9 \\
\hline Panel 297 (MG) & 96.4 & 85.0 & 73.3 & 58.3 & 44.9 & 45.9 & 27.6 & 65.1 \\
\hline Panel 237 (MG) & 92.3 & 82.8 & 65.9 & 65.9 & 70.1 & 50.1 & 34.8 & 85.9 \\
\hline Panel 126 (MG) & 99.5 & 110.9 & 73.1 & 67.7 & 64.0 & 71.5 & 43.0 & 98.7 \\
\hline Panel 262 (MG) & 99.4 & 106.6 & 95.0 & 73.8 & 81.1 & 74.0 & 50.3 & 98.4 \\
\hline Panel $279(\mathrm{FG})^{\mathrm{h}}$ & -- & 94.6 & 93.2 & 91.8 & 91.6 & $86.7^{\mathrm{i}}$ & 81.9 & 90.7 \\
\hline SRM $1579^{j}$ & - & 100.2 & 106.4 & 109.6 & 95.6 & $92.9^{k}$ & -. & -- \\
\hline ELPAT 032 & -- & 87.9 & 89.3 & 91.4 & 100.1 & $90.7^{k}$ & -- & -- \\
\hline
\end{tabular}

${ }^{a}$ Extraction without ultrasound was performed using a water bath with mechanical stirring of the specimen in the acid solution.

${ }^{\mathrm{b}}$ Extraction with ultrasound was conducted using Sonicator No. 1 .

'Extraction-with-ultrasound results for the MG panels are taken from NISTIR 6571 [7]; other such results are from the current study.

${ }^{\mathrm{d}}$ Low and high temperatures correspond to ranges of $\approx 42{ }^{\circ} \mathrm{C}$ to $47^{\circ} \mathrm{C}$ and $\approx 62{ }^{\circ} \mathrm{C}$ to $67^{\circ} \mathrm{C}$, respectively. Short and long times were $30 \mathrm{~min}$ and $90 \mathrm{~min}$, respectively.

${ }^{\mathrm{e}}$ Rep. indicates replicate determination.

${ }^{\mathrm{f}}$ Mean of 10 measurements unless otherwise indicated.

${ }^{g}$ MG indicates that the specimen was manually ground after cooling in dry ice.

${ }^{\mathrm{h}} \mathrm{FG}$ indicates that the specimen was mechanically ground in a freezer mill at liquid nitrogen temperatures.

${ }^{i}$ Mean of 4 measurements; the range of values and accompanying uncertainties of the measurements are given in Figure 3C.

Some initial measurements for SRM 1579 gave less than $80 \%$. These results could not be replicated.

${ }^{\mathrm{k}}$ Result of a single measurement. 
low temperature and short time. When the extractions without ultrasound were performed at high temperature and short time, the lead recoveries were either within the range of, or greater than, the values attained in the previous NIST study using Sonicator No. 1. Finally, when the extractions without ultrasound were conducted at high temperature and long time, lead recoveries for specimens from five of the six panels (Nos. 126, 141, 237, 262, and 297) were quantitative. This was the same result obtained when specimens from these panels were sonicated using high temperature and long time in the extraction experiment on the series of NIST paint-film panels (Tables 12A and 12B). Only specimens from Panel 127 exhibited less than quantitative recovery $(59 \%)$ using extraction without ultrasound at high temperature and long time. For unexplored reasons, extraction of lead from this panel is apparently difficult. Note in Table 12A, for example, that Panel 127 had a recovery of $65 \%$ when sonicated at high temperature and long time.

Similarly, sonication of freezer-mill ground specimens from Panel 127 in the preliminary testing of factors influencing lead recovery gave recoveries of $\approx 70 \%$.

In summary, this experiment on extraction without ultrasound showed that, for specimens sampled from selected NIST paint-film panels and SRM 1579 and ELPAT 032, sonication is not necessary for lead extraction. These results reflect the findings of Grohse et al. [14], who conducted a similar experiment using "static extraction" (i.e., neither specimen agitation nor ultrasound) of SRM 1579. They reported $>90 \%$ recovery for two out of five SRM 1579 specimens after $3 \mathrm{~h}$ using $25 \%$ $\mathrm{HNO}_{3}$; they did not indicate the temperature. Similarly, Ashley, Andrews, Cavazos and Demange [28] have reported that, under the same acid conditions, extraction of lead from selected SRMs without sonication gave recoveries that were, for the most part, statistically equivalent to those obtained using UE.

3.4 Proposed Conditions for Lead Extraction in the Field. Because this study found significant interactions of particle size with sonication temperature and time, recommendations are provided in Table 16 for extraction of lead from paint-film specimens in the field under temperature and time conditions that are selected based on the particle size of the ground specimen. Note that the recommendations do not address the method of grinding specimens; that is, any procedure suffices whether it is manual grinding with or without dry ice, or mechanically grinding at room temperature or under cryogenic (e.g., liquid $\mathrm{N}_{2}$ ) conditions. If small particles $(\leq 425 \mu \mathrm{m})$ are obtained, then extraction is performed under the relatively convenient temperature and time conditions of $\approx 45^{\circ} \mathrm{C}$ and $30 \mathrm{~min}$ that are incorporated in the procedure for performing an analysis using commercial UE/ASV apparatus. However, as found in the current and previous NIST UE/ASV studies and also in the Ashley et al. [3] field study, some paint-film specimens may be more difficult to grind than others. As a consequence, the sufficiently small particle sizes that allow the relatively convenient temperature and time conditions may not always be realized. In these cases, longer times and/or higher temperatures are recommended.

The recommendations in Table 16 are both practical and conservative. Because they are based on the limited data developed in the current study, they are not optimized for particle size, temperature, and time. Figure 12 was prepared using the particle size data in Table 6 and presents the mass (in $\%$ mass fraction) of the ground specimen that passed a given sieve as a function of mesh size. Note in Figure 12 that $100 \%$ of the freezer-mill ground (i.e., small particle size) specimen from Panel 279 passed a No. 40 sieve (425 $\mu$ m mesh). Recall from Figure 6 that these specimens yielded quantitative lead recovery even when extracted at low temperature and short time (i.e., $42{ }^{\circ} \mathrm{C}$ to $47^{\circ} \mathrm{C}$ and $\left.30 \mathrm{~min}\right)$. Observe also in Figure 12 that essentially all ( $\approx 85 \%$ ) of the mass of specimens from the thin-latex Panel 179, which were manually ground after cooling in dry ice (i.e., large particle size), passed a No. 20 sieve $(850 \mu \mathrm{m}$ mesh $)$. When the series of NIST paint-film panels 
Table 16. Recommendations for extraction temperature and time as related to the particle size of ground laboratory-prepared paint-film specimens

\begin{tabular}{|c|c|c|}
\hline$\frac{\text { Specimen Particle Size }}{\mu \mathrm{m}}$ & $\frac{\text { Particle Size Determination }}{\text { Sieve Analysis }}$ & $\begin{array}{l}\text { Extraction Conditions }^{a} \\
\text { Temperature and Time }\end{array}$ \\
\hline - $\leq 425$ & Particles pass a No. 40 Sieve. & $\approx 45^{\circ} \mathrm{C}$ and $30 \mathrm{~min}$ \\
\hline - $\quad>425$ but $\leq 850$ & $\begin{array}{l}\text { Particles pass a No. } 20 \text { Sieve, but } \\
\text { not a No. } 40 \text { Sieve. }\end{array}$ & $\approx 65^{\circ} \mathrm{C}$ and $90 \mathrm{~min}$ \\
\hline - $>850$ & $\begin{array}{l}\text { Some particles do not pass a No. } 20 \\
\text { Sieve. }\end{array}$ & $\begin{array}{l}\quad \approx 45^{\circ} \mathrm{C} \text { and } 270 \mathrm{~min} \\
\text { or } \\
\text { (in lieu of field extraction, hotplate or } \\
\text { microwave digestion in a laboratory) }\end{array}$ \\
\hline
\end{tabular}

"It is proposed that, as is done in current practice, sonicators be continually used in the field for "agitating" the specimen in the $\mathrm{HNO}_{3}$ solution and for maintaining the extraction temperature. Field-portable sonicators are generally affordable and practical in that they are relatively small and light in weight, and may have temperature control. Moreover, multiple samples may be simultaneously extracted. Use of a sonicator does not rule out other extraction means, e.g., a water bath, for controlling the extraction conditions.

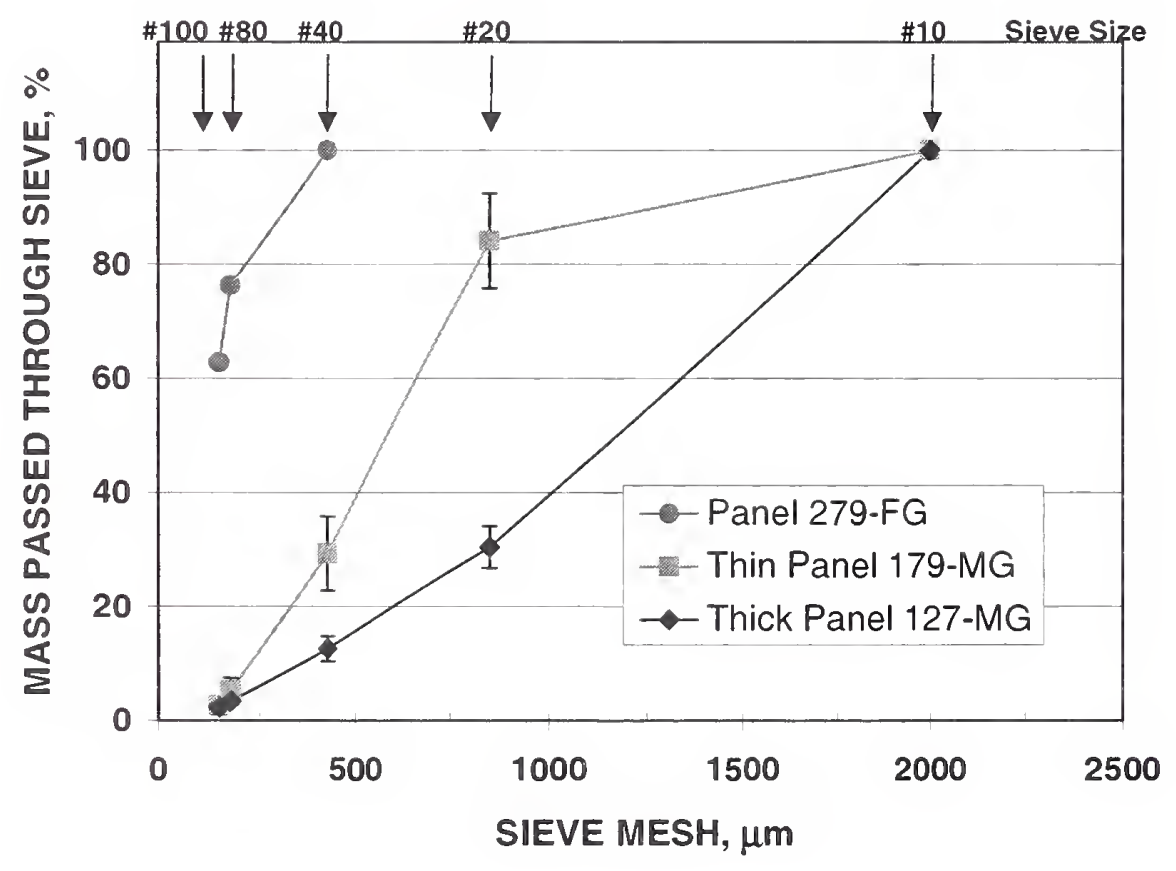

Figure 12. Specimen Mass Passing a Sieve as a Function of Sieve Mesh. FG indicates that the specimen was ground using a freezer mill at liquid nitrogen temperatures; MG indicates that the specimen was manually ground after cooling in dry ice. The error bars are one standard deviation. For Panel 127, the data points are for one measurement only and, thus, error bars are not included. 
(Section 3.2) were subjected to UE/ASV analysis using high temperature and long time (i.e., $62{ }^{\circ} \mathrm{C}$ to $67^{\circ} \mathrm{C}$ and $90 \mathrm{~min}$ ), with one exception, the thin-latex panels provided quantitative recovery (Tables 12A and 12B). In contrast, in the previous NIST UE/ASV study [7], the thin-latex panels provided an average recovery of only $70 \%$. That is, the low temperature and short time used in the previous study was not sufficient for specimens having particle sizes presumably of the order of $425 \mu \mathrm{m}$ to $850 \mu \mathrm{m}$ or greater. Finally, as listed in Tables $12 \mathrm{~A}$ and $12 \mathrm{~B}$, it was found that about $40 \%$ of the thick-oil specimens did not give quantitative recovery even after extraction at high temperature and long time. Note in Figure 12 that only about $30 \%$ of the manually ground specimens sampled from the thick-oil Panel 127 passed through the No. 20 sieve. In contrast, quantitative lead recovery from Panel 127 was achieved at $42{ }^{\circ} \mathrm{C}$ to $47^{\circ} \mathrm{C}$ for $270 \mathrm{~min}$. Finally, as noted in Table 16, if extracting for $270 \mathrm{~min}$ is not acceptable for field application, hotplate or microwave digestion in the laboratory is an alternative.

As previously discussed, manual grinding of the NIST laboratory-prepared paint-film specimens after cooling in dry ice did not achieve particle sizes (e.g., $\leq 425 \mu \mathrm{m}$ ) that would allow for extraction using the relatively convenient conditions of $\approx 45^{\circ} \mathrm{C}$ and $30 \mathrm{~min}$. However, EPA data demonstrate that such small particle sizes can be achieved with manual grinding of "real-world" paint-film specimens. For example, Hodson et al. [18] reported that specimens manually ground after cooling in dry ice displayed particle sizes from $1.2 \mu \mathrm{m}$ to $120 \mu \mathrm{m}$ or $150 \mu \mathrm{m}$. Similarly, Grohse et al. [14] indicated that typical paint specimens passed through a No. 60 or a No. 80 sieve (250 $\mu \mathrm{m}$ and $180 \mu \mathrm{m}$ mesh, respectively) after grinding. Presumably Grohse et al. [14] used manual grinding, as they indicated that "a simple $30 \mathrm{~s}$ crushing operation with a glass or plastic rod appears adequate." Likewise, Harper and Gutknecht [21] have indicated that manual mortar and pestle grinding for at least $1.5 \mathrm{~min}$ provided similar particle size distributions; for example, in the case of samples taken from a wooden cabinet door, the particle sizes ranged from $\approx 1 \mu \mathrm{m}$ to $200 \mu \mathrm{m}$.

For estimating particle sizes in the field, it is not recommended to sieve the ground specimen that is to be subjected to ASV lead analysis. Sieving the specimen may be prone to specimen loss or contamination. The UE/ASV operator should first visually compare the particle size of the ground specimen with that of a previously ground paint film known to have a particle size of less than $425 \mu \mathrm{m}$. In cases where paint-film specimens are readily ground to a visually apparent fine powder, the UE/ASV analysis can proceed using the relatively convenient temperature and time extraction conditions of $45^{\circ} \mathrm{C}$ and $30 \mathrm{~min}$. If any question arises that the particle size of the ground specimen is not adequately small, then a second paint specimen should be sampled from the location adjacent to the first specimen. After grinding, this specimen should be sieved using No. 20 and No. 40 sieves. The results of this surrogate sieving should be taken as an estimate of the particle size of the original specimen and the extraction conditions for the original specimen should be based on the recommendations given in Table 16.

As final comments on the recommendations given in Table 16, note that continued use of sonicators for lead extraction in the field is suggested, although data in this study indicate that comparable lead recoveries are obtained with and without ultrasound. Use of field-portable sonicators, as is done in current practice, provides a practical and generally affordable means of "agitating" [14] and heating specimens during lead extraction in the field. Small sonicators are available that have heating capability. In addition, it is noted that the recommendations in Table 16 are based on the results of UE/ASV testing using laboratory-prepared paint film specimens. The recommendations have not been examined in field testing using specimens taken from housing. Such testing will be the subject of future study. 


\section{SUMMARY, CONCLUSIONS, AND RECOMMENDATIONS}

In 2001, NIST reported on a study investigating the reliability of field-portable UE/ASV for quantitatively determining lead in laboratory-prepared paint films when certified lead inspectors trained to conduct UE/ASV tests performed the analyses. A main finding was that lead levels determined according to the UE/ASV protocol were often considerably less than the amount of lead in the laboratory-prepared paint films. Limited data suggested that an important contributor was incomplete lead solubilization during ultrasonication of the paint-film specimens. Understanding why the UE method used in this NIST study apparently resulted in low lead recovery is important to using ASV in the field. Other than UE, common extraction methods such as hotplate or microwave acid digestion are not suitable for routine field use. Consequently, a follow-up laboratory study was performed to examine factors affecting the ultrasonic extraction of lead from laboratory-prepared paint specimens.

The study had three phases that were, for the most part, performed sequentially. In Phase I, an investigation was performed to identify factors affecting ultrasonic extraction of lead from laboratory-prepared paint films. In a preliminary experiment, an examination of sonicator power was undertaken on the presumption that higher power might provide greater lead recovery. The sonicator used in the previous NIST UE/ASV study had $45 \mathrm{~W}$ power, and it was questioned whether this was sufficient for acceptable lead extraction. Thus, extractions were carried out using nine sonicators having power ranging from $45 \mathrm{~W}$ to $833 \mathrm{~W}$. In the main Phase I experiment, five variables - sonicator power, specimen mass, specimen particle size, sonication temperature, and sonication time-were systematically examined in a two-level full-factorial experiment. "Low" $(45 \mathrm{~W})$ and "high" ( $600 \mathrm{~W})$ power sonicators were used in the extraction of manually ground ("large particle size") and freezer-mill ground ("small particle size") specimens having either "small" (25 mg) or "large" (100 mg) mass. Sonication was conducted at "high" $\left(62{ }^{\circ} \mathrm{C}\right.$ to $\left.67^{\circ} \mathrm{C}\right)$ or "low" $\left(42^{\circ} \mathrm{C}\right.$ to $47^{\circ} \mathrm{C}$ ) temperatures for "long" ( $90 \mathrm{~min}$ ) or "short" (30 min) times.

In Phase II, ultrasonic extraction, conducted under sonication temperature and time conditions found in Phase I to enhance lead recovery, was performed on specimens sampled from each of the same 80 laboratory-prepared paint-film panels that were used in the previous NIST UE/ASV study. These panels included lead-containing paint films prepared with either white lead or lead chromate pigments mixed in an oil-based paint. For each pigment type, these panels had 10 lead levels ranging from $0 \mathrm{mg} / \mathrm{cm}^{2}$ to $3.5 \mathrm{mg} / \mathrm{cm}^{2}$. The paint films were adhered to steel or plaster substrates, which were a priori considered to be difficult or easy to sample, respectively. Overlayers covering the lead-based films were either a relatively thickly applied oil-based paint $(0.75 \mathrm{~mm}$ to $1.4 \mathrm{~mm})$, or a relatively thinly applied latex paint $(0.13 \mathrm{~mm}$ to $0.28 \mathrm{~mm})$.

In Phase III, lead extractions from specimens suinpled from a limited number of the NIST paintfilm panels, and also from reference material samples were performed without ultrasound using a water bath with mechanical stirring of the specimen in the acid solution. The results were compared with those obtained when extraction was conducted using a sonicator. Based on the results of the three phases of the study, recommendations were proposed for extraction of lead from paint-film specimens in the field under varying temperature and time conditions as a function of specimen particle size. 
The main conclusions of the study are:

- No significant effect of sonicator power on lead recovery was found for specimens extracted at $42{ }^{\circ} \mathrm{C}$ to $47^{\circ} \mathrm{C}$ for 30 min, even though sonicator power ranged from $45 \mathrm{~W}$ to $833 \mathrm{~W}$.

- Three significant main effects—small particle size, high temperature, and long time-were found in the five-factor experiment. Two significant two-way interactions-particle size with temperature and particle size with time-were also observed. No three-way interactions were found. Small particle size, high temperature, and long time were $\leq 425 \mu \mathrm{m}, 62{ }^{\circ} \mathrm{C}$ to $67{ }^{\circ} \mathrm{C}$, and 90 min, respectively.

- The effect of particle size on lead recovery was quite strong-when the particle size was small, mean lead recovery was quantitative regardless of the conditions of sonication temperature and time. In contrast, when the particle size was large, only in the case of high temperature and long time was the mean recovery quantitative.

- Lead recovery from manually ground (i.e., large particle size) specimens sampled from the 80 NIST laboratory-prepared paint-film panels and extracted at high temperature and long time (i.e., $62{ }^{\circ} \mathrm{C}$ to $67^{\circ} \mathrm{C}$ for $90 \mathrm{~min}$ ) was always greater than the mean recoveries determined for such specimens in the previous NIST UE/ASV study using low temperature and short time (i.e., $42{ }^{\circ} \mathrm{C}$ to $47^{\circ} \mathrm{C}$ for $30 \mathrm{~min}$ ).

- Specimens sampled from the paint-film panels in the previous NIST UE/ASV study were generally not ground to a sufficiently small particle size to allow quantitative extraction under the temperature and time conditions used for extraction.

- Lead extractions of specimens from a limited number of the NIST paint-film panels, and also from reference material samples, gave comparable lead recoveries when performed with and without ultrasound under the same conditions of temperature and time.

- The main role of the sonicator in UE extraction of lead from paint-film specimens is apparently to "agitate" the specimen in the acid solution, and to provide a source of heating.

The recommendations from the study, which were developed based on tests using laboratoryprepared paint film specimens and which will be the subject of future field testing, are:

- When conducting UE/ASV analysis of a paint-film specimen in the field, the particle size of ground specimen should be estimated and an appropriate temperature and time condition for lead extraction should then be selected. Proposed combinations of extraction temperatures and times as a function of particle size are:

\begin{tabular}{|c|c|c|}
\hline Particle Size, $\mu \mathrm{m}$ & Sieve Analysis & Extraction Temperature and Time \\
\hline$\leq 425$ & Passes a No. 40 sieve & $\approx 45^{\circ} \mathrm{C}$ and $30 \mathrm{~min}$ \\
\hline$>425$ but $\leq 850$ & $\begin{array}{l}\text { Passes a No. } 20 \text { sieve, } \\
\text { but not a No. } 40 \text { sieve }\end{array}$ & $\approx 65^{\circ} \mathrm{C}$ and $90 \mathrm{~min}$ \\
\hline$>850$ & $\begin{array}{l}\text { Does not pass a No. } 20 \\
\text { sieve }\end{array}$ & $\begin{array}{l}\approx 45^{\circ} \mathrm{C} \text { and } 270 \mathrm{~min} \\
\text { (alternatively, hotplate or } \\
\text { microwave digestion) }\end{array}$ \\
\hline
\end{tabular}

For small particle size $(\leq 425 \mu \mathrm{m})$, the proposed combination of extraction temperature and time is the same as that incorporated in current practice for conducting an UE/ASV analysis using commercial apparatus. 
- The particle size of a ground specimen should only be estimated in the field using sieving when it is apparent that the test specimen is not finely ground. In this regard, the UE/ASV operator should visually compare the particle size of the ground specimen with that of a previously ground paint film (or other similar substance such as ground black pepper) known to have a particle size of less than $425 \mu \mathrm{m}$. In cases where paint-film specimens are readily ground to a visually apparent fine powder, the UE/ASV analysis can proceed using the relatively convenient temperature and time extraction conditions of $45^{\circ} \mathrm{C}$ and $30 \mathrm{~min}$. If the particle size of the ground specimen does not appear adequately small, then a second paint specimen should be sampled from the location adjacent to the first specimen. After grinding, this specimen should be sieved. The results of this surrogate sieving should be taken as an estimate of the particle size of the original specimen and the extraction conditions for the original specimen should be based on the recommendations given above.

- Continued use of sonicators is acceptable for lead extraction in the field, as they provide for a practical and generally affordable means of agitating and heating specimens.

\section{ACKNOWLEDGMENTS}

The U. S. Department of Housing and Urban Development (HUD) sponsored this study. The authors appreciate the support and encouragement of Peter Ashley, Warren Friedman, and David Jacobs of the HUD Office of Healthy Homes and Lead Hazard Control. The authors acknowledge with thanks the noteworthy contributions of those many individuals who assisted in the study. Kevin Ashley, CDC National Institute for Occupational Safety and Health; Gary Dewalt, Quantech; William Gutknecht, Research Triangle Institute; Sharon Harper, EPA National Exposure Research Laboratory; and Richard Harris, NIST Building and Fire Research Laboratory, provided NIST research staff use of their sonicators. NIST colleagues, Steven Fick and Gerald Blessing, discussed sonicator operation and gave timely review and comment on the conduct of the study. Kevin Ashley and Sharon Harper, as well as NIST colleagues, Joannie Chin, Steven Fick, Jonathan Martin, and Tinh Nguyen, provided critical reviews of this report.

\section{REFERENCES}

[1] Ashley, K., "Ultrasonic Extraction and Field-Portable Anodic Stripping Voltammetry of Lead from Environmental Samples, Electroanalysis, Vol. 7, No. 12 (1995), pp. 1189-1192.

[2] Williams, E.E., Van Hise, C.C., and Gutknecht, W.F., "Evaluation of the Performance of Reflectance and Electrochemical Technologies for the Measurements of Lead in Characterized Paints, Bulk Dusts, and Soils," EPA 600/R-95/003, Environmental Protection Agency, Research Triangle Park (April 1996).

[3] Ashley, K., Hunter, M., Tait, L.H., Dozier, J., Seaman, J.L., and Berry, P.F., "Field Evaluation of On-Site Techniques for the Measurement of Lead in Paint Films," Field Analytical Chemistry and Technology, Vol. 2, No. 1 (1998), pp. 39-50.

[4] Ashley, K., "On-Site Extraction and Anodic Stripping Voltammetric Determination of Lead," Woebkenberg, Mary Lynn, Ed., Applied Occupational and Environmental Hygiene, Vol. 13, No. 2 (February 1998), pp. 94-98.

[5] Ashley, K., Mapp, K.J., and Millson, M., "Ultrasonic Extraction and Field-Portable Anodic Stripping Voltammetry for the Determination of Lead in Workplace Air Samples," American Industrial Hygiene Association Journal, Vol. 59 (October 1998), pp. 671-679. 
[6] Ashley, K., Song, R., Esche, C.A., Schlecht, P.C., Baron, P.A., and Wise, T.J., "Ultrasonic Extraction and Portable Anodic Stripping Voltammetric Measurement of Lead in Paint, Dust Wipes, Soil, and Air: An Interlaboratory Evaluation," Journal of Environmental Monitoring, Vol. 1 (1999), pp. 459-464.

[7] Rossiter, W.J., Jr., Vangel, M.G., McKnight, M.E., Signor, A., and Byrd, W. E., "Ultrasonic Extraction/Anodic Stripping Voltammetry for Determining Lead in Household Paint: A Laboratory Evaluation," NISTIR 6571, National Institute of Standards and Technology, Gaithersburg, MD (May 2001), 66 pages.

[8] Rossiter, W.J., Jr., Vangel, M.G., McKnight, M.E., and Dewalt, G., "'Spot Test Kits For Detecting Lead in Household Paint: A Laboratory Evaluation," NISTIR 6398, National Institute of Standards and Technology, Gaithersburg, MD (March 2000), 97 pages.

[9] "Evaluating Performance of On-Site Extraction and Field-Portable Electrochemical or Spectrophotometric Analysis for Lead," ASTM Standard Guide E 1775, Annual Book of Standards, Vol. 04.07, American Society for Testing and Materials, West Conshohocken, PA (2000).

[10] Grohse, P.M., Gutknecht, W.F., Luk, K.K., Wilson, B.M., and Van Hise, C.C., "Standard Operating Procedure for Laboratory Analysis of Lead in Paint, Bulk Dust, and Soil by Ultrasonic, Acid Digestion and Inductively Coupled Plasma Emission Spectrometric Measurement," EPA 600/R-95/111 (September 1997), 36 pages.

[11] Luk, K.K., Grohse, P.M., Hodson, L.L., Binstock, D.A., Van Hise, C.C., and Gutknecht, W.F., "Standard Operating Procedure for Field Analysis of Lead in Paint, Bulk Dust, and Soil by Ultrasonic, Acid Digestion and Colorimetric Measurement," EPA 600/R-93/200 (September 1993), 34 pages.

[12] "Ultrasonic Extraction of Paint, Dust, Soil, and Air Samples for Subsequent Determination of Lead," ASTM Standard Practice E 1979, Annual Book of Standards, Vol. 04.11, American Society for Testing and Materials, West Conshohocken, PA (2001).

[13] "Wire Cloth and Sieves for Testing Purposes," ASTM Standard Specification E 11, Annual Book of Standards, Vol. 14.02, American Society for Testing and Materials, West Conshohocken, PA (2001).

[14] Grohse, P.M., Luk, K.K., Hodson, L.L., Wilson, B.M., Gutknecht, W.F., Harper, S.L., Beard, M.E., Lim, B.S., and Breen, J.J., "Development of a Field-Test Method for the Determination of Lead in Paint and Paint-Contaminated Dust and Soil," Chapter 20, Lead Poisoning: Exposure, Abatement, Regulation, Eds., J.J. Breen and C.R. Stoup, CRC Press, Lewis Publishers, Boca Raton (1995), pp. 161-168.

[15] Certificate of Analysis, Standard Reference Material 1579, "Powdered Lead Based Paint," National Institute of Standards and Technology, Gaithersburg, MD (January 23, 1973).

[16] Mason, T.J. and Lorimer, J.P., Sonochemistry: Theory, Applications, and Uses of Ultrasound in Chemistry, John Wiley \& Sons, New York, NY (1988). 
[17] Ashley, K., Wise, T.J., Mercado, W., and Parry, D.B., "Ultrasonic Extraction and FieldPortable Anodic Stripping Voltammetric Measurement of Lead in Dust Wipe Samples," Journal of Hazardous Materials, Vol. 83, No. 1 (2001), pp. 41-50.

[18] Hodson, L.L., Hardison, E.D., Leinbach, A.A., Messner, M.J., Binstock, D.A., and Gutknecht, W.F., "An Intercomparison of Grinding Techniques Used for the Preparation of Lead-in-Paint Samples, EPA 600/R-95/112 (September 1997), 79 pages.

[19] Amoedo, L., Capelo, J.L., Lavilla, I., and Bendicho, C., "Ulrasound-Assisted Extraction of Lead from Solid Samples: a New Perspective on the Slurry-Based Sample Preparation Methods for Electrothermal Atomic Absorption Spectrometry," Journal of Analytical Atomic Spectrometry, Vol. 14 (1999), pp. 1221-1226.

[20] Nascentes, C.C., Korn, M., and Arruda, M.A.Z., “A Fast Ultrasound-Assisted Extraction of Ca, Mg, Mn, and Zn from Vegetables," Microchemical Journal, Vol. 69 (2001), pp. 37-43.

[21] Harper, S.L. and Gutknecht, W.F., "Sources of Variability in Collection and Preparation of Paint and Lead-Coating Samples," Journal of Environmental Monitoring, Vol. 3 (2001), 335-340.

[22] Shoh, A., "Industrial Applications of Ultrasound," Chapter 3 in Ultrasound: Its Clienical, Physical, and Biological Effects, Suslick, K.S., Ed., VCH Publishers, New York, NY (1988), pp. 97-122.

[23] Mason, T.J., Practical Sonochemistry: Users Guide to Applications in Chemistry and Chenical Engineering, Ellis Horwood, New York, NY (1991).

[24] Suslick, K.S., Ed., Ultrasound: Its Chemical, Physical, and Biological Effects, VCH Publishers, New York, NY (1988).

[25] Luche, J., Synthetic Organic Sonochemistry, Plenum Press, New York, NY (1998).

[26] Feigl, F. and Anger, V., Spot Tests in Inorganic Analysis, Elsevier Publishing Co., New York, NY (1972), p. 250.

[27] Crank, J., Tlee Mathematics of Diffusion, Oxford Press (1975).

[28] Ashley, K., Andrews, R.N., Cavazos, L., and Demange, M., "Ultrasonic Extraction as a Sample Preparation Technique for Elemental Analysis by Atomic Spectrometry," Journal of Analytical Atomic Spectrometry, Vol. 16 (2001), pp. 1147-1153. 

